University of Warwick institutional repository: http://go.warwick.ac.uk/wrap This paper is made available online in accordance with publisher policies. Please scroll down to view the document itself. Please refer to the repository record for this item and our policy information available from the repository home page for further information.

To see the final version of this paper please visit the publisher's website. Access to the published version may require a subscription.

Author(s): J.-D. FOURNIER and S. GALTIER

Article Title: Meromorphy and topology of localized solutions in the Thomas-MHD model

Year of publication: 2001

Link to published version:

http://dx.doi.org/10.1017/S002237780100887X

Publisher statement: None 


\title{
Meromorphy and topology of localized solutions in the Thomas-MHD model
}

\author{
J. -D. FOUR IER ${ }^{1}$ and S. GA LTIE R $\mathrm{R}^{1,2,3}$ \\ ${ }^{1}$ Department G.D. Cassini, Observatoire de la Côte d'Azur and CNRS, BP 4229, \\ 06304 Nice Cedex 4, France \\ ${ }^{2}$ Mathematics Institute, University of Warwick, Coventry, CV4 7AL, UK \\ ${ }^{3}$ Present and permanent address: Institut d'Astrophysique Spatiale, Université Paris XI, \\ 91405 Orsay Cedex, France
}

(Received 1 December 1999 and in revised form 17 March 2000)

\begin{abstract}
The one-dimensional MHD system first introduced by J.H. Thomas [Phys. Fluids 11, 1245 (1968)] as a model of the dynamo effect is thoroughly studied in the limit of large magnetic Prandtl number. The focus is on two types of localized solutions involving shocks (antishocks) and hollow (bump) waves. Numerical simulations suggest phenomenological rules concerning their generation, stability and basin of attraction. Their topology, amplitude and thickness are compared favourably with those of the meromorphic travelling waves, which are obtained exactly, and respectively those of asymptotic descriptions involving rational or degenerate elliptic functions. The meromorphy bars the existence of certain configurations, while others are explained by assuming imaginary residues. These explanations are tested using the numerical amplitude and phase of the Fourier transforms as probes of the analyticity properties. Theoretically, the proof of the partial integrability backs up the role ascribed to meromorphy. Practically, predictions are derived for MHD plasmas.
\end{abstract}

\section{Introduction}

In the present theoretical work, the reader should not expect the physical modelling of any particular plasma - natural or experimental. Following the other route, we start from a well-known particular one-dimensional magnetohydrodynamic (1DMHD) theory, the Thomas (1968) system, and we derive its predictions. Using elementary dynamical systems theory, asymptotic and complex analysis, and precise numerical simulations, we derive exact results and reliable phenomenological laws on intermittency, energy balance and integrability. We first discuss the domain of validity of the various hypotheses made in obtaining the Thomas system and in the course of its study.

\subsection{Aim and scope of this study}

Our motivation is to contribute to a better understanding of certain phenomena occurring in natural plasmas where the 1D-MHD level of description is sufficient. It is now widely recognized that the MHD approximation is adequate for a variety of astrophysical and geophysical plasmas (Parker 1994), ranging from the Sun (Priest 1982; Galsgaard and Nordlund 1996) and the solar wind (Dobrowolny et al. 1980; Zank and Matthaeus 1992; Marsch and Tu 1994, Goldstein and Roberts 1999) to 
the interstellar medium (Heiles et al. 1993; Bhattacharjee et al. 1998). The MHD approximation is in turn a convenient tool for a thorough study of very anisotropic plasmas (Shebalin et al. 1983; Ng and Bhattacharjee 1997; Galtier et al. 2000) - not to mention laboratory plasmas created in tokamaks (Wild et al. 1981; Taylor 1986).

Specifically, the phenomena we have in mind are shocks (Festou et al. 1993; Zilbersher et al. 1998; Pouquet et al. 1999) and localized oscillations, as well as the spatio-temporal intermittency events present in the interstellar medium and in the solar corona, or more generally in the heliosphere (Burlaga 1991; Schwenn and Marsch 1991), of which solar flares are but a catastrophic example (Galtier and Pouquet 1998); here the $1 \mathrm{D}$ reduction proves to be rich enough to capture the physics and mathematics of the phenomena. On the other hand, in some other cases, this reduction is the last resort, given the formidable task presented both mathematically and numerically by the full 3D-MHD problem. It is especially so if one is to perform a large number (of large duration) of computer simulations to obtain reliable statistics, which is in order given the turbulence present in most of the media cited above. Our experience (Galtier and Pouquet 1998) is that 1D models are useful and rewarding given the non-trivial physical effects they can embody and the quality of the statistics. Of course, for a good global understanding, the statistical approach has to be complemented and clarified by the prior study of the deterministic structures (cf. Galtier and Fournier (1998), where a preliminary account of the present work was given) and singularities generated in the flow by the nonlinear dynamics.

Finally, we focus our study on situations where the ratio of the kinematic viscosity and the magnetic resistivity, the magnetic Prandtl number, is large, as encountered, for example, in the interstellar medium (Heiles et al. 1993). Conversely our study predicts a preference for states where the magnetic energy is larger than the kinetic one - a situation encountered in some regions of the heliosphere (Schwenn and Marsch 1991); according to our findings, this could be a hint that the Prandtl number is large in these regions. For the mathematical part of our study, the Prandtl number is taken to be infinite; that is, the magnetic resistivity is set to zero.

With these guidelines in mind, we consider in the present paper the localized (in space) solutions and the integrability properties of a particular $1 \mathrm{D}$ model of MHD; the model is akin to those used in previous statistical and deterministic studies (Galtier and Pouquet 1998); up to this point, our study is entirely deterministic. We derive exact solutions of the travelling-wave problem, which are meromorphic (in space), as well as meromorphic (in space) approximations of localized solutions of the genuine time-dependent problem. We study numerically the stability of localised (or 'topological') solutions and the validity of their meromorphic approximations. Incidentally, we hope to convince the reader (i) how worthwhile it is to go over to the complex plane (Chabat 1990; Flaschka et al. 1991) to detect and characterise hidden singularities (Fournier and Bessis 1994) that govern the shape, the integrability and the dynamics of localized real solutions (Fournier 1986); (ii) that partial integrability (see Conte and Boccara 1990; see the end of this section and Sec. 5) gives a handle on features and (quasi-) solutions of certain nonlinear systems.

\subsection{The Thomas-MHD model}

The particular 1D MHD model that we study is the one introduced by Thomas (1968) as a model of the dynamo effect. It is given by

$$
\partial_{t} v+v \partial_{x} v=b \partial_{x} b+\sigma \partial_{x x} v
$$




$$
\partial_{t} b+v \partial_{x} b=b \partial_{x} v+\eta \partial_{x x} b
$$

where $v(x, t)$ is the velocity field, $b(x, t)$ the magnetic induction, $\sigma$ the kinematic viscosity and $\eta$ the magnetic resistivity. We want to mention that these equations, under various disguises, represent various physical phenomena. For instance, in the inviscid limit, the system (1.1) is conjugate to the system

$$
\begin{gathered}
\partial_{t} v+v \partial_{x} v=-\frac{1}{\gamma M^{2}} \frac{1}{\rho} \partial_{x} \rho^{\gamma}, \\
\partial_{t} \rho+\partial_{x}(\rho v)=0,
\end{gathered}
$$

under the mapping

$$
b=\frac{1}{M} \rho^{(\gamma-1) / 2},
$$

and the restriction $\gamma=-1$ (Thomas 1970). The system (1.2) describes the dynamics of polytropic gases with index $\gamma$, density $\rho$ and Mach number $M$. The physical, hydrodynamical and mathematical aspects of this system or systems akin to it have been extensively studied in the literature. Brunelli and Das (1997) have obtained a Lax representation of the system (1.2) for $\gamma \in \mathbb{N}^{*}$. Olver and Nutku (1988) have exhibited the Hamiltonian structure of a family of hydrodynamic type, including (1.2), but the value $\gamma=-1$ appears as a singular case. Statistical investigations have been pursued recently by Passot and Vázquez-Semadeni (1998), with emphasis on the symmetric role of the case $\gamma=1$.

Going back to the MHD problem, we stress that the system (1.1) has the advantages of having the same nonlinearities as the full MHD equations and also the same form for the dissipative effects. The system is characterized by two dimensionless numbers: the Reynolds number $\mathscr{R}_{e}$, which is proportional to $1 / \sigma$, and the magnetic Prandtl number

$$
P_{M}=\frac{\sigma}{\eta} .
$$

The main difference from the full MHD equations is that the solenoidal constraint has been dropped both for $v$ and $b$; its presence would of course completely trivialize this 1D problem. Here, in the opposite case, we have a sort of infinite compressibility, but this phenomenon is balanced by dissipative effects. A similar discussion could be given with regard to the Burgers equation, of which the Thomas model (1.1) is the most natural generalization to the magnetic case. We just note here in passing that after the exciting introduction of his equation by Burgers (1939) (and see also Burgers 1974) as a model of turbulence, the discovery (Hopf 1950; Cole 1951) that it could be linearized depreciated it in his own view! Nevertheless the Burgers equation is still today a rich source of inspiration, a physical model and a mathematical toy; the structures and the singularities that it produces are actively studied in both the deterministic and statistical cases (Kida 1979; Fournier and Frisch 1983; Bessis and Fournier 1984, 1990; Gurbatov et al. 1997). We believe that, with the aid of the accumulated experience on the Burgers equation, the Thomas model is worth similar efforts.

We recall that in the inviscid limit $\left(\sigma \rightarrow 0^{+}\right)$, the Burgers equation is singular in the real domain: the solution exhibits 'true' shocks, with a finite discontinuity of the velocity. When a coupled magnetic induction is introduced, as in (1.1) but with zero diffusion term $(\eta=0)$, the singularity is prevented in strong initial magnetic induction regions and favoured by neutral points. This was proved by Sulem et al. 
(1979) for the Thomas model with $\sigma=\eta=0$, using real analysis tools. We shall refer later to their results as governing asymptotic regimes.

In a more recent study, Passot and Pouquet (1986) have discussed the integrability of the model on the basis of the nature of the complex singularities, the so-called Painlevé test (Ablowitz et al. 1980; Weiss et al. 1983; Passot 1986). They pointed to the possibility that in subcases (e.g. $\eta=0$ ) the system (possibly endowed with an additional integrability constraint) could be solved, although in most cases the system should have infinitely multivalued singularities; generically, this entails a clustering of identically singular points, which precludes integrability. It is an instance of partial integrability, a concept whose validity has since been widely recognised (Levine and Tabor 1988; Fournier et al. 1989; Conte and Boccara 1990) and has motivated us to look for exact solutions in the zero-magnetic-resistivity case. In an unpublished study, Passot (1987) also conducted numerical experiments at moderate resolution ( 64 modes) with magnetic Prandtl number and Reynolds number ranging typically from 1 to 10 . In several instances, he observed localized profiles for $v, b$ and/or the reduced Elsässer variables $z^{ \pm}=v \pm b$. Those could take the form of fronts, pulses or hollow waves, which were all reminiscent of limiting cases of Jacobi elliptic functions. We therefore considered it a challenge to find meromorphic solutions or at least prove the existence of nearby complex poles whose position, motion, order and residue would explain these shapes.

\section{Travelling waves at infinite Prandtl number : exact results}

By infinite Prandtl number, we mean here zero magnetic resistivity $\eta$.

\subsection{Profiles of the travelling waves}

We study hereinafter solutions $\{\tilde{v}(x, t), \tilde{b}(x, t)\}$ of the system (1.1) that depend only on the reduced variable $X=x-c t$. In applying a Galilean transform

$$
\begin{gathered}
v=\tilde{v}-c, \\
b=\tilde{b},
\end{gathered}
$$

it is clear that the profiles $v(X)$ and $b(X)$ of these travelling waves are solutions of the stationary Thomas system (1.1). We rewrite it hereinafter for convenience as

$$
\begin{gathered}
v v^{\prime}=b b^{\prime}+\sigma v^{\prime \prime}, \\
v b^{\prime}-b v^{\prime}=0,
\end{gathered}
$$

where the primes indicate derivatives with respect to $X$. The system (2.2) is invariant under space translation; all the profiles obtained here should be understood as functions of $X-X_{*}$, where $X_{*}$ is a free, real or possibly complex number (since the solutions can be analytically continued).

We first consider the case of zero kinematic viscosity, where the solutions are either (i) a pure constant for both $v$ and $b$ or (ii) a free function of $X$ provided that $v$ and $b$ are equal up to a sign. The latter seems to have curiosity value, but we stress that in this case the nonlinearities in $(2.2 \mathrm{a})$ annihilate each other.

Turning to $\sigma>0$, we mention the possibility of an identically vanishing velocity field, with which a pure constant magnetic induction is associated; and of an identically vanishing magnetic induction, which maps back the problem to the Burgers equation. 
Finally, there is again here the possibility of $v$ and $b$ having the same modulus, but this time the presence of the kinematic viscosity restricts the solutions to a constant or affine behaviour.

Once these extreme cases have been examined, $(2.2 \mathrm{~b})$ indicates that the ratio of the two fields is a non-degenerate pure constant. Precisely, we note that

$$
b=D v
$$

with $D$ being a finite real number (since $v$ and $b$ are real for $X$ real) but with -1 , 0 and 1 excluded. Using then (2.3) in (2.2a) and integrating once, one gets

$$
\frac{1}{2}\left(1-D^{2}\right) v^{2}+\lambda=\sigma v^{\prime},
$$

where $\lambda$ is a free integration constant belonging to $\mathbb{R}$. Three different regimes must now be studied according to the position of the product $\lambda\left(D^{2}-1\right)$ with respect to 0 .

If $\lambda$ vanishes, (2.4) can be trivially integrated, leading to the solution

$$
\begin{aligned}
& v=-2 \sigma \frac{1}{1-D^{2}} \frac{1}{X}, \\
& b=-2 \sigma \frac{D}{1-D^{2}} \frac{1}{X} .
\end{aligned}
$$

If $\lambda\left(D^{2}-1\right)$ is negative, a simple change of variable and of function,

$$
v=\mathscr{A} \tan [\varphi(X)]
$$

leads to the solution

$$
\begin{gathered}
v=\mathscr{A} \tan \left(\frac{\mathscr{A}}{2 \sigma}\left(1-D^{2}\right) X\right), \\
b=D v .
\end{gathered}
$$

The amplitude $\mathscr{A}$ is a free real positive number. The freedom in the choice of $\mathscr{A}$ stands for the freedom of the constant previously denoted by $\lambda$.

If $\lambda\left(D^{2}-1\right)$ is positive, the solutions are no longer circular but rather hyperbolic functions. Depending on the sign of the derivative, one gets respectively

$$
\begin{gathered}
v=-\mathscr{A} \operatorname{coth}\left(\frac{\mathscr{A}}{2 \sigma}\left(1-D^{2}\right) X\right), \\
v=\text { constant, } \\
v=-\mathscr{A} \tanh \left(\frac{\mathscr{A}}{2 \sigma}\left(1-D^{2}\right) X\right) ;
\end{gathered}
$$

in each case,

$$
b=D v .
$$

We note in passing that, on using a complex translation, as discussed above, with

$$
X_{*}=i \frac{\pi}{2} \frac{2 \sigma}{\mathscr{A}\left(1-D^{2}\right)},
$$

the solution $(2.8 \mathrm{c})$ produces the solution $(2.8 \mathrm{a})$. 


\subsection{Singularity analysis}

At the end of this rather elementary but systematic discussion, we want to stress that all non-trivial solutions are meromorphic functions exhibiting one or an infinite number of poles in the real or complex domain. Of course, the singularities of $v$ and $b$ have the same position. Also, the reality of the fields for real $X$ implies that each singularity in the complex plane is accompanied by a singularity with the complexconjugate position and a complex-conjugate amplitude factor. We shall not enter into a discussion of the convergence of the Laurent series or of the questions of their formal existence or the possibility of logarithmic corrections; the last two points will be the subject of Sec. 5. Of course, for the travelling-wave solutions obtained above, all of this information can be read off from (2.5)-(2.8). Nevertheless, we want to show how indications on the nature of singularities and the amplitude factors can be obtained from the structure of the travelling-wave equations (2.2), using the so-called dominant singularity analysis. Let us suppose that locally (somewhere in the complex plane)

$$
\begin{aligned}
& v \simeq V_{0} X^{-\alpha}, \\
& b \simeq B_{0} X^{-\beta},
\end{aligned}
$$

where $V_{0}$ and $B_{0}$ are (possibly complex) amplitudes, and $\alpha$ and $\beta$ are, to start with, positive rational numbers. Using this local behaviour in $(2.2 \mathrm{~b})$ implies equality between $\alpha$ and $\beta$. Going then to (2.2a), the nonlinear terms are of the same order of magnitude, and the question is to compare this with the magnitude of the dissipative term. Of course, it can only be of the same order or subdominant. In the latter case, the amplitudes $V_{0}$ and $B_{0}$ have also to be equal up to a sign. Now, we know from $(2.2 \mathrm{~b})$ and $(2.3)$ that the ratio of the two fields is a pure constant. So, if $V_{0}^{2}=B_{0}^{2}$, this means that $|v|=|b|$ globally - a particular case already studied and where solutions are entire functions. Thus each of the three terms in (2.2a) contributes to the dominant singularity analysis; this implies that $\alpha=\beta=1$, which is a simple pole; for the moment, we assume they are pure poles (e.g. no logarithmic corrections). For the amplitudes (residues) the singularity analysis of (2.2a) implies

$$
V_{0}^{2}=B_{0}^{2}-2 \sigma V_{0} .
$$

In contrast to the Burgers equation, the residues are not constrained by the structure of the equations. In reference to the Burgers case, we introduce normalized residues such that $V_{0}=-2 \sigma W$ and $B_{0}=-2 \sigma Z$. These normalized residues are a priori free complex numbers depending on the considered pole of $v$ (respectively $b$ ). However, for a given pole, they cannot be chosen independently. They are related by

$$
Z^{2}=W^{2}-W .
$$

It is noteworthy that, for any given travelling-wave solution, the residues are real and identical for all the poles. Given the fact that the ratio $D$ of the two fields is a pure constant, it is thus also the case for the ratio of the residues $-2 \sigma Z$ and $-2 \sigma W$. The ratio of the normalized residues is in turn equal to $D$; given (2.12), one thus has

$$
\begin{aligned}
& W=\frac{1}{1-D^{2}}, \\
& Z=\frac{D}{1-D^{2}},
\end{aligned}
$$


as is simply seen from (2.5) or (2.8). The dominant singularity analysis and the relation (2.12) extend to the possible meromorphic solutions of the time-dependent problem, since the terms with time derivative in (1.1) do not contribute to the dominant singularity analysis of these equations. This will be of some importance in Secs 3 and 4, and will be discussed thoroughly in Sec. 5 .

\subsection{Phase space and orbits}

For completeness, and to mention a link with the previous remarks, we want here to present the study of the geometrical aspect of the problem (2.2) with a strictly positive viscosity. Here no claim is made for originality (see e.g. Arnold 1973), but we need to go into details for the announced discussion. Again we put aside the regimes where the ratio $D=b / v$ is infinite or plus or minus one. Under this restriction, the Thomas system reduces to the non-trivial regimes of (2.2a). This family of second-order differential equations, for the profile $v$ as a function of $X$, is parametrized by $D$. Given the sign of $1-D^{2}$, it can be mapped into a unique dynamical system via the following change of variables

$$
\begin{gathered}
s=\frac{\left|1-D^{2}\right|}{\sigma} X, \\
\xi_{1}(s)=v(X), \\
\xi_{2}(s)=\frac{\sigma}{\left|1-D^{2}\right|} v^{\prime}(X) .
\end{gathered}
$$

The phase space of this two-dimensional system will be the plane $\left(\xi_{1}, \xi_{2}\right)$, and the dynamical equations then read

$$
\begin{gathered}
\frac{d \xi_{1}}{d s}=\dot{\xi}_{1}=\xi_{2}, \\
\frac{d \xi_{2}}{d s}=\dot{\xi}_{2}=\operatorname{sign}\left(1-D^{2}\right) \xi_{1} \xi_{2},
\end{gathered}
$$

where $\left(\dot{\xi}_{1}, \dot{\xi}_{2}\right)$ is the velocity vector field of the dynamical system. We note at once that changing the sign of $1-D^{2}$ is just equivalent to considering a new vector field obtained from the previous one by symmetry with respect to the $\xi_{1}$ axis, an operation that keeps unchanged the topology of the field. From now on, we shall thus restrict our study to $D^{2}<1$. In doing so, we are actually studying the analogous dynamical system associated with the stationary Burgers equation with a viscosity equal to one.

We proceed to the local analysis of the field (2.15) (see Fig. 1). All the points of the $\xi_{1}$ axis are fixed points, and there are no others in the finite domain. These points are thus neutral in the $\xi_{1}$ direction. The differential flow of the field at any point of the $\xi_{1}$ axis exhibits an eigenvector $\mathbf{e}=\left(1, \xi_{1}\right)$ associated with an eigenvalue equal to $\xi_{1}$. The point at infinity is also a fixed point. This can be seen using inverse polar coordinates; by this, we mean rewriting the vector field (2.15) in the plane $(\rho, \theta)$, where $\theta$ is the polar angle and $\rho$ the inverse modulus of the vector $\left(\xi_{1}, \xi_{2}\right)$. In this reference system, the point $\left(0, \frac{1}{2} \pi\right)$ is indeed a fixed point. We note in addition that on the $\xi_{2}$ axis, the field is parallel to the $\xi_{1}$ axis, with an intensity such that it reaches the first bisecting line $\left(\xi_{2}=\xi_{1}\right)$, under identification of the phase space and its tangent space.

We turn now to global analysis. As we know from the previous section, the 


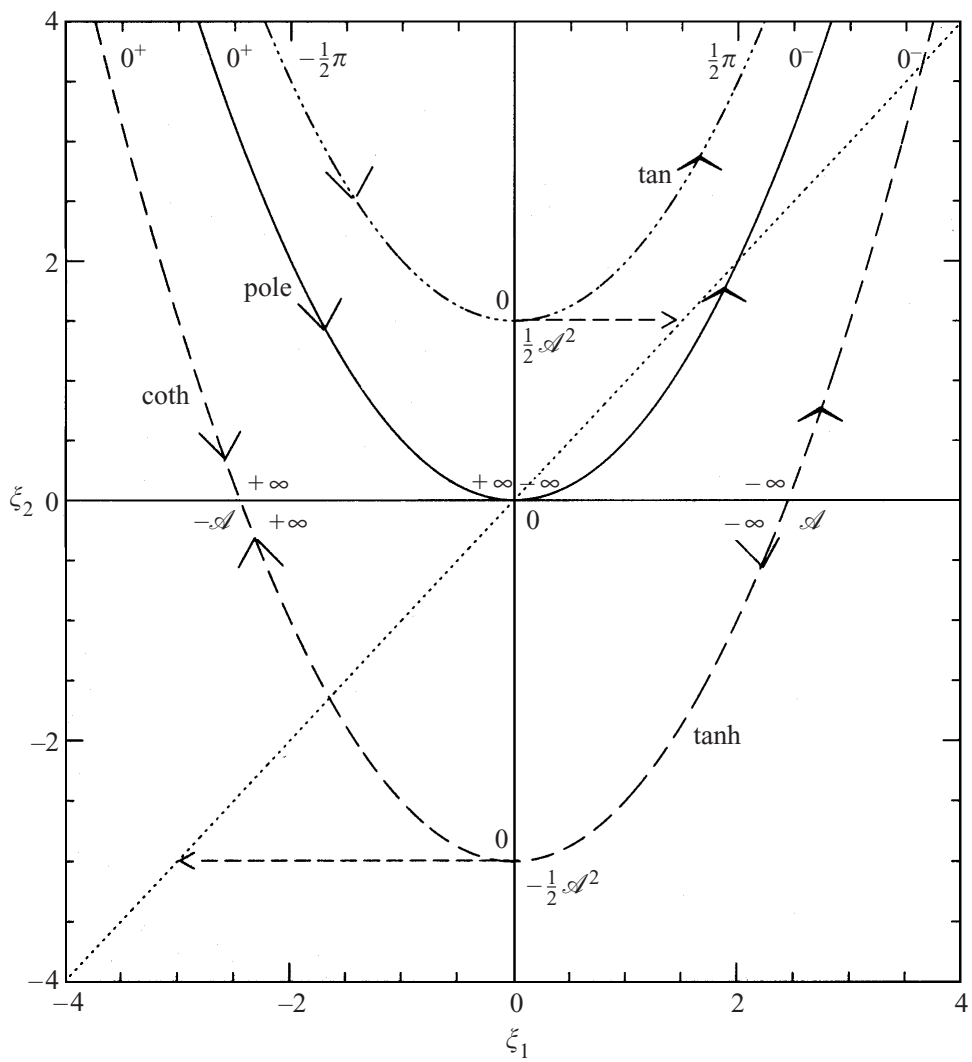

Figure 1. Phase-space representation of the dynamical system constituted by the stationary Thomas system in the normalized coordinates $\left(\xi_{1}, \xi_{2}\right)$. All the points of the $\xi_{1}$ axis are fixed points; on the $\xi_{2}$ axis, the field is parallel to the $\xi_{1}$ axis, with an intensity (represented by the arrows) that reaches the first bisecting line. The integral lines of the system correspond to three different classes of parabolas. The arches of these parabolas correspond to the solutions (2.5)-(2.8). The tan solution corresponds to the upper parabola (dash-dotted line), the unique pole solution to the middle one (solid line), the coth solution to the two upper-half-plane branches of the lower one (dashed line) and the tanh solution to the lower half-plane branch of the same parabola. The phase points travel according to the arrows. The parabolas are labelled: inside by the time and outside by the values of $\xi_{1}$ and $\xi_{2}$ when finite.

differential system (2.2) can be integrated. In terms of the associated dynamical system, this means that we can write down the equation of the integral lines, namely

$$
\xi_{2}=\frac{1}{2} \xi_{1}^{2}+\mu .
$$

This family of parabolas divides into three classes (Fig. 1). For positive $\mu$, the parabola is entirely in the upper half-plane, and, in keeping with the previous notation, we write

$$
\xi_{2}=\frac{1}{2} \xi_{1}^{2}+\frac{1}{2} \mathscr{A}^{2}
$$

A limiting case is obtained for $\mu=0$. For negative $\mu$, the parabola equation reads

$$
\xi_{2}=\frac{1}{2} \xi_{1}^{2}-\frac{1}{2} \mathscr{A}^{2} \text {. }
$$

It crosses the $\xi_{1}$ axis, and thus divides into three branches.

Combining the geometrical information (2.16) and the dynamical content of (2.15a) yields exactly (2.4), which has already been solved and discussed. The ad- 
vantage of the point of view of this section is that a topological meaning is attached to each of the four families of solutions. The tan solutions (2.7) correspond to the upper-half-plane parabola; the representative phase point travels from the upper left corner down to the summit of the parabola and then to the upper right corner, as times elapses from $\left(-\frac{1}{2} \pi\right)$ to $\left(\frac{1}{2} \pi\right)$. The unique pole solution $(2.5)$ corresponds to the limiting case $\mu=0$. Here the phase point travels from the origin of the plane to the upper right corner, as time elapses from minus infinity to zero; then it comes back from the point at infinity, down the upper left branch, and approaches the origin as time goes to infinity. The coth solutions $(2.8 \mathrm{a}, \mathrm{d})$ correspond to the two upper-half-plane branches of the parabolas, whose summit lies in the lower halfplane; the phase point starts, for infinite negative times, from a point $(\mathscr{A}, 0)$; it is bound to the point at infinity in the upper right corner and comes back from infinity via the upper left corner, to reach, as time goes to infinity, the point $(-\mathscr{A}, 0)$. Finally the tanh solutions $(2.8 \mathrm{c}, \mathrm{d})$ correspond to the lower branch of the previous parabolas; starting from $(\mathscr{A}, 0)$ at large negative times, the phase point reaches the summit $\left(0,-\mathscr{A}^{2}\right)$ at time equal to zero and the symmetric point $(-\mathscr{A}, 0)$ at large positive times.

The first comment is that what we called 'non-trivial' solutions of (2.2) in Sec. 2.2 are all heteroclinic orbits of the system (2.15), joining two fixed points, or three in the coth case. It is a fact of life that travelling-wave solutions of partially integrable PDEs are often meromorphic-or at least those that correspond to heteroclinic orbits have this property. Our second comment is that in the present case, all nontrivial travelling waves are heteroclinic orbits and do correspond to meromorphic solutions.

\subsection{Realistic solutions}

We discuss now the feature of the physically relevant solutions - that is, those that are real for $X$ real and have no singularity in the real domain. Apart from the trivial solutions, this means that we study the profiles $(2.8 \mathrm{c}, \mathrm{d})$, which we rewrite here for convenience:

$$
\begin{gathered}
v=-\mathscr{A} \tanh \left(\frac{\mathscr{A}}{2 \sigma}\left(1-D^{2}\right) X\right), \\
b=-D \mathscr{A} \tanh \left(\frac{\mathscr{A}}{2 \sigma}\left(1-D^{2}\right) X\right) ;
\end{gathered}
$$

we recall that $\mathscr{A}$ is a strictly positive real number and $D$ is a real number such that $D\left(1-D^{2}\right)$ does not vanish. For the velocity field, the jump across the transient region located nearby the origin is $\left[-\mathscr{A} \operatorname{sgn}\left(1-D^{2}\right)\right]$; if it is negative, we call this region a shock, as usual; if it is positive, we call it an antishock. We use the same terminology for the magnetic field.

The richness of this MHD problem appears here in the fact that, in contrast to the Burgers case, the sign of the jump of the profiles (2.19) is not prescribed by the equations. Both $v$ and $b$ may undergo a negative or positive jump depending on the sign of $1-D^{2}$ (respectively $D\left(1-D^{2}\right)$ ), which we call for this reason topological factors. In his unpublished finding, Passot had found one of the configurations generated by the model - the one where both $v$ and $b$ have a shock profile. Three other configurations are clearly possible with $v$ and/or $b$ exhibiting an antishock profile. In all these cases, transient profiles match two plateaus of the physical 
fields; following condensed matter physics terminology, we shall therefore call these solutions defects and the transient region the core of the defect.

One interesting physical prediction of the configurations described by (2.19) concerns the width of the shock (and/or antishock). The jump is always different for $v$ and $b$, but the thickness governed by the argument of the tanh function is the same for both fields. Thus the physics of these regimes, in the absence of magnetic resistivity, is governed by the kinematic viscosity for both fields.

The notion of thickness may seem a priori a bit loose, although there is no doubt about its scaling with respect to the viscosity, the amplitude of the jump and the absolute value of the topological factor $1-D^{2}$. We propose hereinafter two quantitative definitions.

The notion of a shock is naturally associated with the idea of a rapid jump and thus with the presence of a peak in the derivative. From a physicist's point of view, the thickness of the shock can be defined as the spatial extension of the region where the derivative is large; it provides a natural length scale. Let us denote by $M_{d}$ the absolute value of the extremum of the derivative, reached at $X=0$; we define the thickness of the shock (or antishock) by $\delta_{p}$ such that

$$
\left|v^{\prime}\left(\frac{1}{2} \delta_{p}\right)\right|=\frac{M_{d}}{p}
$$

where $p$ is some arbitrary number imposing a definite change in the order of magnitude of the derivative (e.g. $p=10$ ). Using (2.19a), this means that

$$
\delta_{p}=2 \operatorname{arcosh}(\sqrt{p}) \frac{2 \sigma}{\mathscr{A}\left|1-D^{2}\right|} .
$$

Applying the same reasoning to the magnetic induction leads to exactly the same formula. For the range of values of $p$ that is of interest here, the 'arcosh' function varies slowly (like a $\log$ ), and thus the precise choice of $p$ has a weak effect on the definition of the thickness. Taking $p=10$ as an example, we get

$$
\delta_{p}=(3.64 \ldots) \frac{2 \sigma}{\mathscr{A}\left|1-D^{2}\right|} .
$$

A more mathematically oriented alternative definition is to use the analytic continuation of the tanh function. The abrupt change in the real domain can be seen as resulting from the presence of a parade of an infinite number of poles, regularly spaced on the imaginary axis 'above' the shock (or antishock). This point of view also offers a natural length scale, but in the complex domain: the distance to the real axis of the closest pole, i.e. the width of the analyticity strip. Clearly the position $i \delta$ of this pole is reached when the argument of the tanh function is $\frac{1}{2} i \pi$; that is, for

$$
\delta=\frac{\pi}{2} \frac{2 \sigma}{\mathscr{A}\left|1-D^{2}\right|} .
$$

This second definition is quantitatively comparable to the first, but has no arbitrariness. It brings to the fore the fact that the thickness is the same for $v$ and $b$, but depends on both jumps. Its generalization to the time-dependent case will be much used in the next section. 


\section{The dynamically forbidden, admissible and preferred configuration of the shock defects}

The findings of the preceding section take their full value in view of the fact that the predicted shock/antishock configurations are indeed dynamically generated by the time-dependent model, as will be seen in the present section. Although the possibility of four configurations appears as a richness of the model, as we have already pointed out, it is actually remarkable that, by (2.19), it rules out four other configurations. Indeed if one combines the topological idea of the sign of the jump for each field with the quantitative idea of a dominant kinetic or magnetic energy, one can imagine only four topologies but eight configurations. The question thus naturally arises of what happens if one chooses as initial condition one of the four configurations that are not amenable to (2.19). We have actually investigated the dynamical problem for each of the eight possible configurations taken as initial conditions.

Up to this point, we have not obtained any explicit genuine time-dependent global solution, although the likely existence of meromorphic ones is encouraging. This and related questions are discussed in Secs 3.2 and 5. We thus analyse here especially the data at times of order one, when the nonlinear effects have taken place and where a fair local comparison with the explicit travelling-wave solutions of Sec. 2 is possible.

The results in the present section have been obtained from numerical experiments, about which we give now some details. The computation is based on a pseudospectral method that implies periodic boundary conditions; we use a second-order Crank-Nicholson algorithm for the time stepping; a resolution of 2048 grid points is reached; the choice of a magnetic Prandtl number of $10^{3}$ is made, with $\sigma=10^{-2}$ and $\eta=10^{-5}$; the two initial fields and their gradients being of order one, we take a timestep $\Delta T=4 \times 10^{-4}$. Concerning the initial conditions, we know from the Burgers equation that the region with large negative gradients will generate the shocks. Since we are interested in the dynamics of isolated shocks, we choose initial conditions such that only one shock (antishock) per unit length is generated for each field in the body of the cell $[0,2 \pi]$. Others may be generated at the boundaries, and very smoothed shocks and oscillations may be present in between. One could use the spatial periodicity of the numerical solutions in studying both the centre and the edge of the cell for each run. In this presentation, we prefer to concentrate on the centre of the cell, with a larger collection of initial conditions. Also, our study of the travelling waves suggests strongly the dynamical importance of the ratio $D$ of the two fields. In the end, this led us to choose

$$
\begin{aligned}
& u(x, 0)=\mathscr{U}_{0} \sin x, \\
& b(x, 0)=\mathscr{B}_{0} \sin x,
\end{aligned}
$$

where $\mathscr{U}_{0}$ and $\mathscr{B}_{0}$ are real quantities of order one. We avoid initial conditions leading to a trivial problem $\left(\mathscr{U}_{0}=0\right)$ or mapping back the problem to the pure Burgers case $\left(\mathscr{B}_{0}=0\right)$ or giving raise to a quasilinear evolution $\left(\mathscr{B}_{0}= \pm \mathscr{U}_{0}\right)$. Depending on the position of the ratio $\mathscr{D}_{0}=\mathscr{B}_{0} / \mathscr{U}_{0}$ with respect to $+1,0,-1$, and the sign of $\mathscr{U}_{0}$, this opens the possibility of eight different types of initial conditions. In the inviscid non-magnetic case, an initial condition such as (3.1a) would produce a real singularity at $t=1$ and $x=\pi$. It is on this space-time region that we focus our 
attention. An advantage of the choice (3.1) is to include the case studied by Passot $\left(\mathscr{U}_{0}=1\right.$ and $\left.\mathscr{B}_{0}=0.2\right)$.

\subsection{The fate of the eight configurations at times of order one}

To implement the announced programme, we have used the initial conditions (3.1) with $\mathscr{U}_{0}$ and $\mathscr{B}_{0}$ both taken from the set $\{-1,-0.2,+0.2,+1\}$, with all non-trivial combinations being explored. We present here the fate at times of order one of these eight different initial configurations. To discuss our results, we now need a bit of nomenclature; this is done first according to the position of the decreasing real number $\mathscr{D}_{0}$ with respect to $+1,0,-1$, leading to the types $1,2,3$ and 4 ; and then according to the sign of the real number $\mathscr{U}_{0}$, leading to the subtypes $\alpha$ when $\mathscr{U}_{0}>0$, that is, when the velocity has its most negative gradients for $x \simeq \pi$; and to the subtypes $\beta$ in the opposite case $\left(\mathscr{U}_{0}<0\right)$. The configurations described by $(2.19)$ fall into the subtypes $(1 . \beta),(2 . \alpha),(3 . \alpha)$ and $(4 . \beta)$. They will be called 'admissible' configurations in the rest of this section. Without prejudice regarding the results of the numerical exploration of the dynamics, we shall call 'forbidden' the other configurations that do not exist in the travelling-wave problem. The same names are used to characterize the solutions at subsequent times; in the latter case, this is of course only in reference to the local profiles for $x \simeq \pi$. These names are written out with the numerical profiles gathered in Figs 2 and 3. Clearly this study and this classification are redundant because of the invariance of the dynamics of the Thomas model under the symmetry $(v, b) \longrightarrow(v,-b)$. This can be seen on

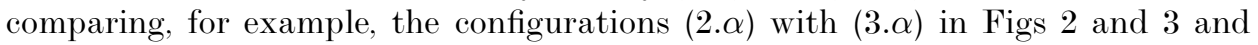
in Table 1. We use the redundancy to present a complete picture and to test our numerical code.

The evolution of initial conditions of types $1(\alpha, \beta)$ and $2(\alpha, \beta)$ are presented in Fig. 2; the drawings depict the profiles of the velocity (dashed line) and of the magnetic induction (dash-dotted line) at the initial time (left-hand panels) and at a later time of order one (right-hand panels). Figure 3 is similar, with initial conditions of types $3(\alpha, \beta)$ and $4(\alpha, \beta)$.

A first look at the figures will show that, at times of order one, the two fields reach, in most cases, a profile reminiscent, at least locally, of the exact travelling-wave solutions of Sec. 2.4. Whenever possible, we have thus fitted the numerical results, at a given time, by the analytic expression (2.19), where $X$ should be understood as $X-\pi$. Clearly from $(2.19)$, the ratio $D$ of the fields is also the ratio of their gradients; for the best construction of the fit, we evaluate $D$ as the numerical value of the ratio of the gradients taken at the core of the defect. In a second step, we obtain a numerical evaluation of $\mathscr{A}$ based on the numerical value of the velocity gradient at the same point.

We shall hereinafter present our findings mainly by commenting upon these fig-

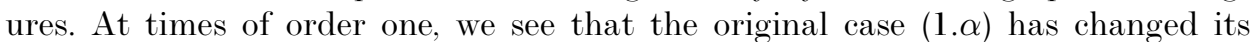
topology, generating a (very smoothed) antishock for $v$, while $b$ generates, as could be expected, a (very smoothed) shock. This new topology is obtained very quickly; the configuration is one of the admissible ones (4. $\beta$ ) and the fits are good locally. Case (1. $\beta$ ) keeps its initial topology, with a (very smoothed) antishock for both $v$ and $b$, and the fits obtained are even better than in the previous case. Case $(2 . \alpha)$ generates the two expected shocks, which can be well fitted by the 'tanh' profiles. In contrast to the previous cases, the forbidden configuration $(2 . \beta)$ does not evolve, after times of order one, towards one of the admissible subcases: like (1. $\beta$ ), the topol- 

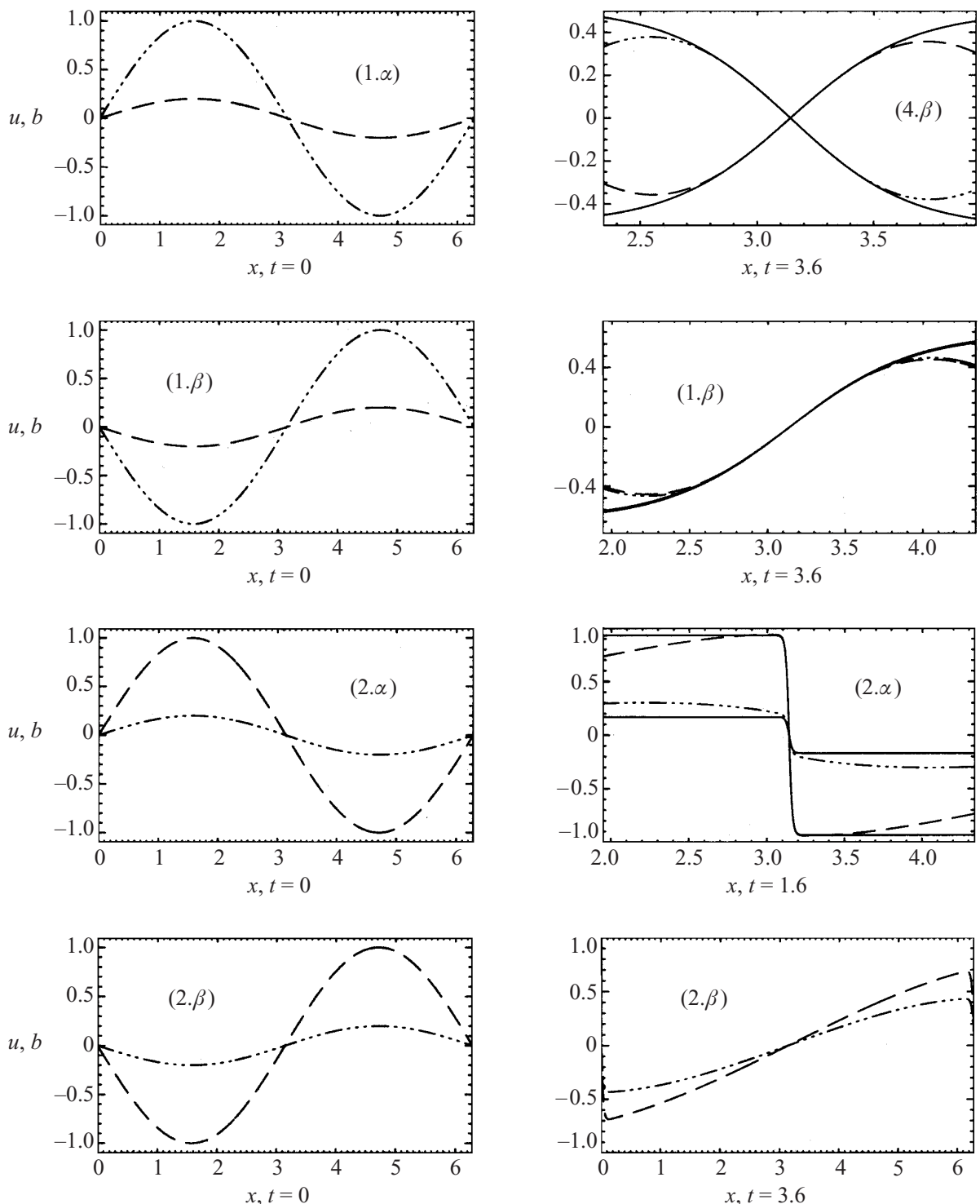

Figure 2. Profiles of the velocity (dashed lines) and the magnetic induction (dash-dotted lines) for the four initial cases $1(\alpha, \beta)$ and $2(\alpha, \beta)$ (left-hand panels); the profiles at times of order one $(t=1.6$ or $t=3.6)$ are depicted in the right-hand panels. Local fits (solid lines) of the profiles with the admissible shock/antishock configurations are also displayed.

ogy is the product of two antishocks, but this time the magnetic energy is smaller than the kinetic one. This exotic behaviour will change in the long run, but at this time no fit with the known coupled profiles is possible. In a similar way, Fig. 3 deals with the last four cases. Cases (3. $\alpha)$ and (4. $\beta$ ) generate the expected configurations and are fitted by the analytical profiles - especially well for $(4 . \beta)$. Case $(4 . \alpha)$, like case $(1 . \alpha)$, changes its topology and reaches the configuration (1. $\beta$ ), which again allows a fit. Finally, the (forbidden) configuration (3. $\beta$ ) keeps its configuration; it 

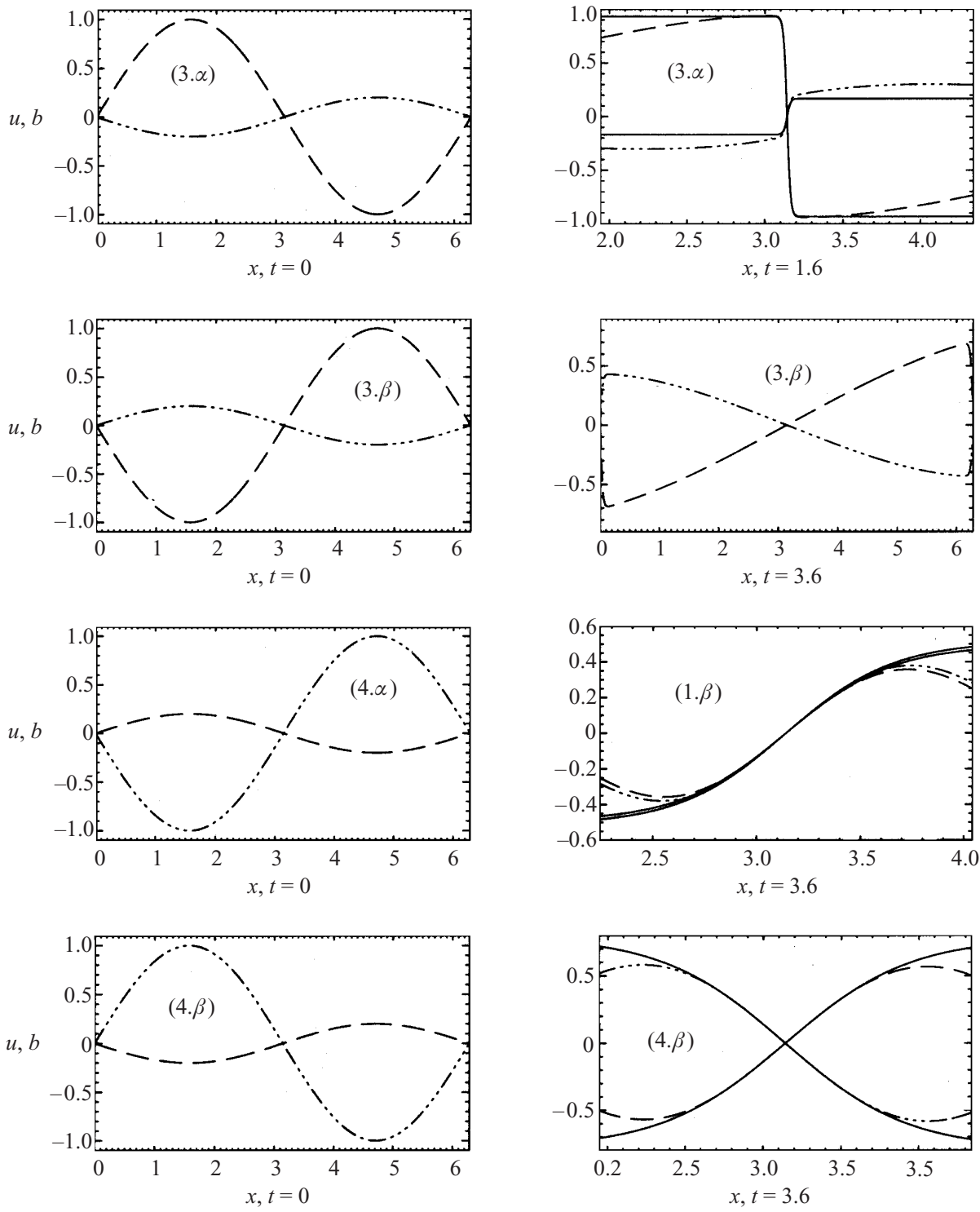

Figure 3. Profiles of the velocity (dashed lines) and the magnetic induction (dash-dotted lines) for the four initial cases $3(\alpha, \beta)$ and $4(\alpha, \beta)$ (left-hand panels); the profiles at times of order one $(t=1.6$ or $t=3.6)$ are depicted in the right-hand panels. Local fits (solid lines) of the profiles with the admissible shock/antishock configurations are also displayed.

has the topology of one of the admissible cases (4. $\beta), v$ antishock/ $b$ shock, but with the 'bad' ratio of magnetic to kinetic energy. Thus no fit is possible with (2.19).

In the light of these results, a first conclusion can be drawn. At times of order one the profiles of the travelling-wave exact solutions provide a good description of the (admissible) configurations (1. $\beta$ ) and (4. $\beta$ ), while for the (admissible) configurations $(2 . \alpha)$ and $(3 . \alpha)$, the validity of the fits is more narrow. This is consistent with the fact that, in the present numerical exploration, the quasishocks (antishocks) of the solutions of types (1. $\beta$ ) and (4. $\beta$ ) exhibit a much greater thickness $\delta$ than those of 


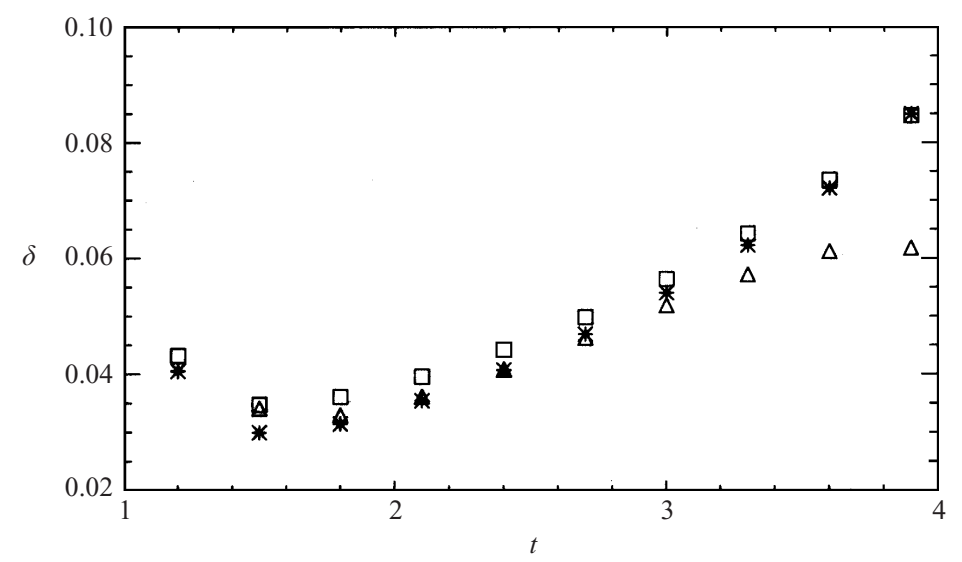

Figure 4. Temporal evolution of the thickness $\delta$ of the shocks created by the original case $(2 . \alpha)$. The value of $\delta$ computed from the relation (2.23) is represented by squares. An advantageous comparison is made with the values of $\delta$ for the shock of the velocity (asterisks) and of the magnetic induction (triangles) deduced from a fit on the spectra of the kinetic energy and of the magnetic energy respectively. In the magnetic case, note the absence of data at $t=1.2$ and the disagreement after $t=3.4$, where no accurate fits are possible.

types (2. $\alpha)$ and (3. $\alpha)$; and, of course, one expects the fits to be correct on a distance $O(\delta)$. A comment on the nonlinear character of the solutions is in order here. The above discussion relies mainly on the fits, themselves based on the magnitude of the two fields $v$ and $b$. To understand more fully the character of the solutions, it is necessary to possess a precise evaluation of the thickness of the defect, which is also the distance to the nearest singularity in the complex. The amplitude $\mathscr{A}$ and the distance $\delta$ are related through an equation that also involves the residue $W$ of the complex pole $((2.23)$ and (2.13); see details below; there is an equivalent formulation for $b$ ). In Table 1 , we present for each admissible configuration discussed here the entire collection of the numerical values of these quantities, along with the magnetic residue $Z$ and the ratio $D$ of the two fields. It is striking that the distance $\delta$ is $O\left(10^{-2}\right)$ for the shock/shock configuration (2. $\left.\alpha\right)$ (and its counterpart $(3 . \alpha)$ ), while it is $O(1)$ for the very smoothed shocks (antishocks) of configurations $(1 . \beta)$ and $(4 . \beta)$. The former cases $((2 . \alpha)$ and $(3 . \alpha))$ are thus strongly nonlinear, and this can be understood with the aid of Theorem 4 of Sulem et al. (1979). In the limiting case $\sigma=\eta=0$, these authors proved that, if there exists, in the initial condition, a neutral point $a_{*}\left(b\left(0, a_{*}\right)=0\right)$ satisfying $\partial_{x} u\left(0, a_{*}\right)<-\left|\partial_{x} b\left(0, a_{*}\right)\right|$, then the system develops a real singularity in finite time. Among the eight initial configurations, only $(2 . \alpha)$ and $(3 . \alpha)$ fulfil the condition (at the neutral point $a_{*}=\pi$; as already mentioned, we forget about the boundaries); in our numerical study with small viscosity and very small diffusivity, they produce quasisingular shapes. In the opposite situation, the value of $\delta$ in the cases $(1 . \beta)$ and $(4 . \beta)$ means that here the extreme nonlinearity of the poles has very little influence in practice. Moreover, it is remarkable that, while both being $O(1)$, the two fields $b$ and $v$ have a ratio very close to 1; as we already noticed in Sec. 2.1, this implies a quasilinear regime. We note that, even in this regime, the solution of Sec. 2 provide a good representation of the (extended) core of the defect. Finally, there are other indications that types $(1 . \beta)$ and (4. $\beta$ ) play a special role. It is indeed remarkable that the two forbidden

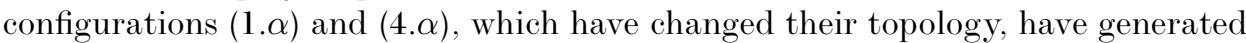


Table 1. Numerical values, for times of order one, of the ratio $D$, the kinetic residue $W$, the magnetic residue $Z$, the amplitude $\mathscr{A}$, the amplitude $D \mathscr{A}$ and the thickness $\delta$ of each of the admissible configurations $(1 . \beta),(2 . \alpha),(3 . \alpha),(4 . \beta)$, described locally by $(2.19)$, reached by the system.

\begin{tabular}{lcccccc}
\hline$t=0$ & $(1 . \alpha)$ & $(1 . \beta)$ & $(2 . \alpha)$ & $(3 . \alpha)$ & $(4 . \alpha)$ & $(4 . \beta)$ \\
$t=O(1)$ & $(4 . \beta)$ & $(1 . \beta)$ & $(2 . \alpha)$ & $(3 . \alpha)$ & $(1 . \beta)$ & $(4 . \beta)$ \\
\hline$D$ & -1.04 & 1.02 & 0.181 & -0.181 & 1.04 & -1.02 \\
$W$ & -12.7 & -31.1 & 1.03 & 1.03 & -12.7 & -31.1 \\
$Z$ & 13.2 & -31.6 & 0.187 & -0.187 & -13.2 & 31.6 \\
$\mathscr{A}$ & 0.494 & 0.782 & 0.933 & 0.933 & 0.495 & 0.782 \\
$D \mathscr{A}$ & -0.514 & 0.798 & 0.169 & -0.169 & 0.515 & 0.789 \\
$\delta$ & 0.807 & 1.25 & 0.035 & 0.035 & 0.807 & 1.25 \\
\hline
\end{tabular}

the configurations (4. $\beta$ ) and (1. $\beta$ ). These latter thus seem to be the more attractive among the admissible configurations. However, a firm conclusion cannot be drawn without knowledge of the fate of the configurations at large times. This will be done in Sec. 3.3.

We return now to the calculation of the thickness $\delta$, concentrating on its timeevolution in the $(2 . \alpha)$ configuration. In section 2.4 , we proposed the relation $(2.23)$ as a possible definition of the thickness $\delta$ of a shock/antishock. The knowledge of the values of $\mathscr{A}$ and $\mathscr{D}$, calculated to obtain at various times fits similar to those presented in Figs 2 and 3, allows us to compute also $\delta$ and to follow its evolution, especially at times of order one. Figure 4 (squares) shows this evolution for an initial condition $(t=0)$ of type $(2 . \alpha)$ (see Fig. 2), with time elapsing from $t=1.2$ to $t=3.9$. We see that the thickness is characterized by a minimum at time $t_{*} \simeq 1.6$, meaning, from the point of view of the complex analysis, that the distance to the real axis of the closest pole is minimal. According to the temporal position with respect to $t_{*}$, two different regimes appear. Before $t_{*}$, the dynamics is mostly nonlinear, with the formation of a shock configuration, the thickness of which decreases to reach a minimum at $t_{*}$; after $t_{*}$, there is a dissipative phase where the thickness of the shock increases.

\subsection{Meromorphy and Fourier transform}

We draw the reader's attention to the mathematical meaning of the calculation of the thickness presented in the previous section. Using (2.23) for this calculation was nothing else than considering the analytic continuation, in the complex spatial domain, of a local real approximation of a numerical profile, by a function of the family (2.19). As already mentioned in Sec. 1, we are actually after the analycity properties of the time-dependent solution itself-which of course are not a priori identical to the analyticity properties of the local fit. A classical mathematical probe of these properties is the behaviour at large wavenumbers of the Fourier transform of the solution. Although, as we have just pointed out, this is a nonrigorous reasoning, we pretend here that the results of Sec. 3.1 and the partial integrability character of the Thomas model are indications of the meromorphy of our time-dependent solutions. We shall also assume that the pattern created by the poles is such that the Fourier integral on $\mathbb{R}$ can be transformed into a contour integral on a curve $\Gamma$ in $\mathbb{C}$ such that (i) there is no pole in the interior of the domain and (ii) the contribution of most of the arcs of $\Gamma$ vanishes when the diameter of the domain goes to infinity. An example of what we mean is given 


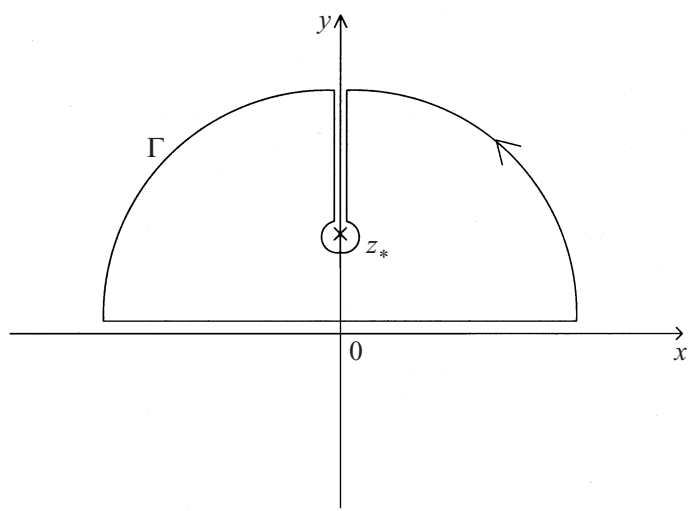

Figure 5. Integration contour $\Gamma$ around the singularity $z_{*}$ in the complex plane.

in Fig. 5. Given these hypotheses, the large wavenumber behaviour of the Fourier transform of the time-dependent solution can be expressed order by order in an asymptotic expansion according to the Laplace method. The main contributions to the Laplace integral come from the arcs of $\Gamma$ that are close to singularities of the solutions. And, apart from pathologies discussed later, the amplitude of the dominant term is controlled by the nature, the amplitude factor and the position of the singularities closest to the real axis. We note in passing that this property was the key technical point in the work by Frisch and Morf (1981) concerning a particular scenario for intermittency in dynamical systems. More relevant to the present work is the paper by Sulem et al. (1983), who, to the best of our knowledge, were the first to make use of the Fourier transform to characterize the analyticity properties of computer-generated solutions of PDEs. They concentrated on the behaviour of the width $\delta(t)$ of the analyticity strip as a function of time; $\delta(t)$ was obtained as the logarithmic decrement of the exponential fall-off of the modulus of the Fourier transform at very large wavenumbers. We have worked along the same lines but with the purpose of extracting more information on the function from the knowledge of its Fourier transform. Specifically, we have examined the modulus and the phase of the overall amplitude factor in front of the dominant term; this factor is indeed generally a complex number that contains information not only on the real part of the position of the singularities but also on the amplitude factors of these singularities. As far as we know, this systematic use of the Fourier transform is a novelty in the context of the numerical study of PDEs. Its value will perhaps be more evident in the following section, but we need to go now into the details.

Let us first consider a meromorphic function $f(z, t)$ with poles of order $n$ (a positive integer); let us consider the pole closest to the real axis, located at $z_{*}(t)=$ $x_{*}(t)+i \delta(t)$. Then the function behaves like

$$
f(z, t) \simeq A\left[z-z_{*}(t)\right]^{-n}
$$

in the neighbourhood of $z_{*}(t)$, where the amplitude factor $A$ is any complex number. As throughout the present work, we assume that $f(z, t)$ is real-analytic, that is, $f(\bar{z}, t)=\overline{f(z, t)}$, where the bar indicates the complex conjugate. Using the techniques described above, we introduce a contour $\Gamma$ that lies entirely in the upper or in the lower complex half-plane. Given our choice of the definition of the Fourier transform integral (see Table 2), the expansion 'à la Laplace' of the integral is then 
Table 2. Some useful Fourier transforms. Note that when needed the Fourier integral is to be understood as a Cauchy principal value integral.

\begin{tabular}{ll}
\hline$f(x)$ & $\hat{f}(k)=\int_{-\infty}^{+\infty} f(x) e^{-i k x} d x$ \\
\hline$\left(1+x^{2}\right)^{-1}$ & $\pi e^{-|k|}$ \\
$x\left(1+x^{2}\right)^{-1}$ & $-\operatorname{sign}(k) i \pi e^{-|k|}$ \\
$\tanh x$ & $-\operatorname{sign}(k) i 2 \pi \cosh \left(\frac{1}{2} k \pi\right) / \cosh (k \pi)$ \\
$1 / \cosh x$ & $\pi / \cosh \left(\frac{1}{2} k \pi\right)$ \\
\hline
\end{tabular}

conveniently done for $k$ negative and large or for $k$ positive and large, the two results being related by Hermitian conjugation. The dominant order of the Fourier transform is thus readily obtained:

$$
\hat{f}(k, t) \simeq 2 \pi \frac{1}{(n-1) !}(-1)^{n} i^{n} A e^{-i k x_{*}(t)} k^{n-1} e^{-k \delta(t)},
$$

for

$$
k \gg 1 \text {. }
$$

A more general and systematic derivation may be found in Frisch and Morf (1981). In the Thomas model, we expect all singularities to be pure simple poles $(n=1)$, as discussed in Sec. 2 (up to possible logarithms - see Sec. 5 - which we overlook in the present regimes). For most of the initial conditions studied here, the abscissa of the defect is $\pi$, and thus one expects that the real part of the location of the singularities will be equal to $\pi$. The resulting asymptotic behaviour of the Fourier transform of the velocity and the magnetic field should thus read

for

$$
\begin{gathered}
\hat{v}(k, t) \simeq(-2 \sigma) W 2 \pi(-i) e^{-i k \pi} e^{-k \delta_{V}(t)}, \\
\hat{b}(k, t) \simeq(-2 \sigma) Z 2 \pi(-i) e^{-i k \pi} e^{-k \delta_{M}(t)},
\end{gathered}
$$

$$
k \gg 1 \text {; }
$$

we have used the normalized residues notation and introduced two different logarithmic decrements $\delta_{V}(t)$ and $\delta_{M}(t)$ for the two fields. From the singularity analysis of (2.2), we have learned that $W$ and $Z$ should be related by (2.12) and that $\delta_{V}(t)$ and $\delta_{M}(t)$ should be equal.

For reasons that will appear immediately, we now turn to the simplest possible case allowed by the hypothesis made on $f(z, t)$, namely a function with only one pair of poles with real identical residues and no additional entire part in the sense of the Mittag-Leffler (Chabat 1990) decomposition. The function $f(z, t)$ then reduces to a rational function, which reads

$$
f(z, t)=(-2 \sigma)(2 \tilde{A}) \frac{z-\pi}{(z-\pi)^{2}+\delta^{2}(t)},
$$

with notation similar to that used in (3.4) and (3.5); in particular, $\tilde{A}$ is the real normalized residue. This extremely simple case has two interesting features. In a real neighbourhood of $\pi$ and over a distance $O(\delta)$, it exhibits a profile reminiscent of a shock (or antishock, depending on the sign of $\tilde{A}$ ) with the maximum gradient located exactly at $\pi$. The Fourier transform of this 'poor man's model' of a shock can be calculated exactly; for positive $k$ it reads

$$
\hat{f}(k, t)=(-2 \sigma) \tilde{A} 2 \pi(-i) e^{-i k \pi} e^{-k \delta(t)}
$$


(we have used Table 2). It is noteworthy that the asymptotic result of the type (3.4), (3.5) is in this simple case an exact representation. Turning now from a rational function to a degenerate elliptic function, one is naturally led to consider the paradigmatic description of a shock by the usual tanh function. Again with the same assumptions and notation, this reads

$$
f(z, t)=(-2 \sigma) \tilde{A} \frac{\pi}{2} \frac{1}{\delta(t)} \tanh \left(\frac{\pi}{2} \frac{z-\pi}{\delta(t)}\right) .
$$

There are now an infinite number of poles with the same (normalized) residue $R$. This gives rise to the plateaus of the tanh function in the real domain, allowing a more global representation of the shock/antishock. This is the representation used in the previous section to fit the shock defect. Again the Fourier transform can be calculated exactly, and reads

$$
\hat{f}(k, t)=(-2 \sigma) \tilde{A}(2 \pi)(-i) \operatorname{sign}(k) e^{-i k \pi} \frac{\cosh [k \delta(t)]}{\cosh [2 k \delta(t)]},
$$

(we have used Table 2). Trivial manipulations of this formula will again produce to the dominant order the formula (3.7).

Going back to the specifics of the numerical simulations, we recall that our numerical scheme produces solutions that are spatially periodic with period $2 \pi$. We are thus dealing with Fourier coefficients with the wavenumber $k$ being an integer, varying from 0 to $\frac{1}{2} N-1$, where $N$ is the resolution (typically, $N=2048$ ). All the above asymptotic considerations on the Fourier integral go over to the case of Fourier coefficients. If at all correct, our description of the shock defects of the Thomas model by tanh profiles thus finally leads to the following predictions concerning the numerical Fourier data:

$$
\begin{gathered}
\text { Phase }[\hat{v}(k, t)]=(-1)^{k} \operatorname{sign}\left(1-D^{2}\right) \frac{\pi}{2} \\
\ln \left[|\hat{v}(k, t)|^{2}\right] \simeq \ln \left(16 \sigma^{2} \pi^{2}\right)-\ln \left[\left(1-D^{2}\right)^{2}\right]-2 \delta_{V}(t) k \\
\text { Phase }[\hat{b}(k, t)]=(-1)^{k} \operatorname{sign}\left[D\left(1-D^{2}\right)\right] \frac{\pi}{2}, \\
\ln \left[|\hat{b}(k, t)|^{2}\right] \simeq \ln \left(16 \sigma^{2} \pi^{2}\right)-\ln \left[\left(\frac{1-D^{2}}{D}\right)^{2}\right]-2 \delta_{M}(t) k
\end{gathered}
$$

for

$$
k \gg 1 \text {. }
$$

We have tested the phase predictions (3.10) and (3.12) for various cases of the classification used in Figs 2 and 3. For times of order one, there is agreement up to the 11th digit between the prediction and the numerics, for practically the whole range of wavenumbers. For the energy spectra the situation is slightly more subtle, as can be seen from Fig. 6. Among all possible configurations, we have chosen to

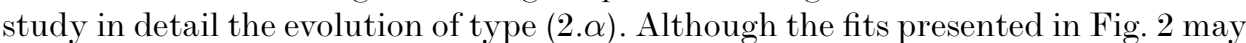
seem discouraging, we point out that the two shocks have a small thickness, which means that the corresponding singularities are close to the real axis, a favourable situation for the type of analysis we perform here. Moreover there is no small-scale excitation outside the core of the defect. In Fig. $6(a)$, the kinetic spectrum $|\hat{v}(k, t)|^{2}$ is plotted on a $\log -\log$ scale as a function of $k$, for various times between $t=1$ and $t=4$. Two comments are in order: for the largest wavenumbers, the numerical 

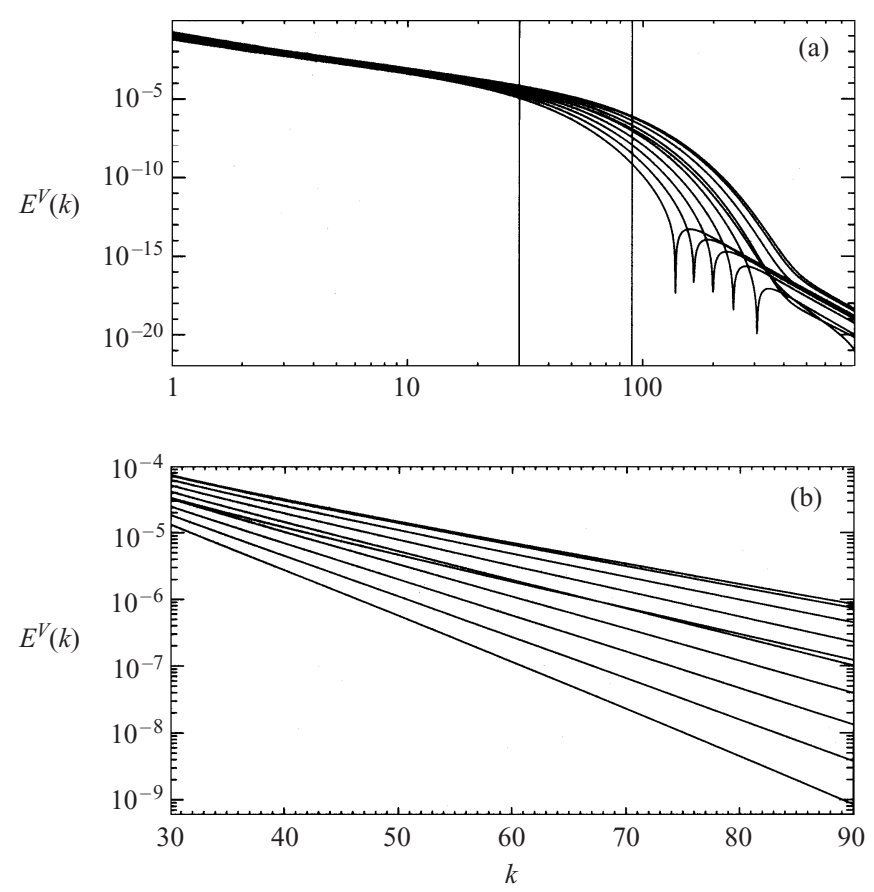

Figure 6. Plots of the kinetic energy spectrum $E^{V}(k)$ for the configuration (2. $\left.\alpha\right)$ (see Fig. 2): (a) on a $\log -\log$ scale; (b) on a linear-log scale. $E^{V}(k)$ is plotted at times between $t=1.2$ and $t=3.9$. The kinetic spectra between $k=30$ and $k=90$ exhibit an exponential fall off ((b), corresponding to the zone between the two vertical lines in (a)).

noise (unavoidable with such small dissipation coefficients) dominates; for slightly smaller wavenumbers, oscillations are clearly visible, which may be attributed to the interaction of singularities whose locations do not have the same real part, a point that we have not pursued. Finally, on decreasing once more the wavenumbers, one enters the range where a distinct exponential fall-off can be seen. Concentrating on the range $k \in[30,90]$ and using a linear-log representation (Fig. 6b), we can fit the spectrum by straight lines, the slope of which is $-2 \delta_{V}(t)$. The same process was followed for the magnetic induction. Both results are plotted in Fig. 4 along with the previous evaluation of the shock thickness based on the fits. We just stress here the good agreement between the three processes.

\subsection{Large-time states: stability and metastability of the admissible configurations}

The numerical experiments described in the introduction to this section have been carried out for times up to many hundreds. We present and discuss here the fate at moderate times and large times of the eight different initial configurations.

At moderate times, two interesting phenomena appear for configurations (2. $\beta$ ) and (3. $\beta$ ). We recall that at times of order one, the initial configuration (2. $\beta$ ) had kept its topology, while the ratio of the magnetic energy to the kinetic energy had grown from 0.2 to almost 1; this tendency continues, and eventually the energetic ratio crosses the critical value 1 . According to our classification, one has thus reached the configuration (1. $\beta$ ), which is one of the four admissible configurations. The same applies for (3. $\beta$ ), which eventually reaches an admissible state of type (4. $\beta$ ). We now comment that, more or less rapidly, all eight possible initial configur- 


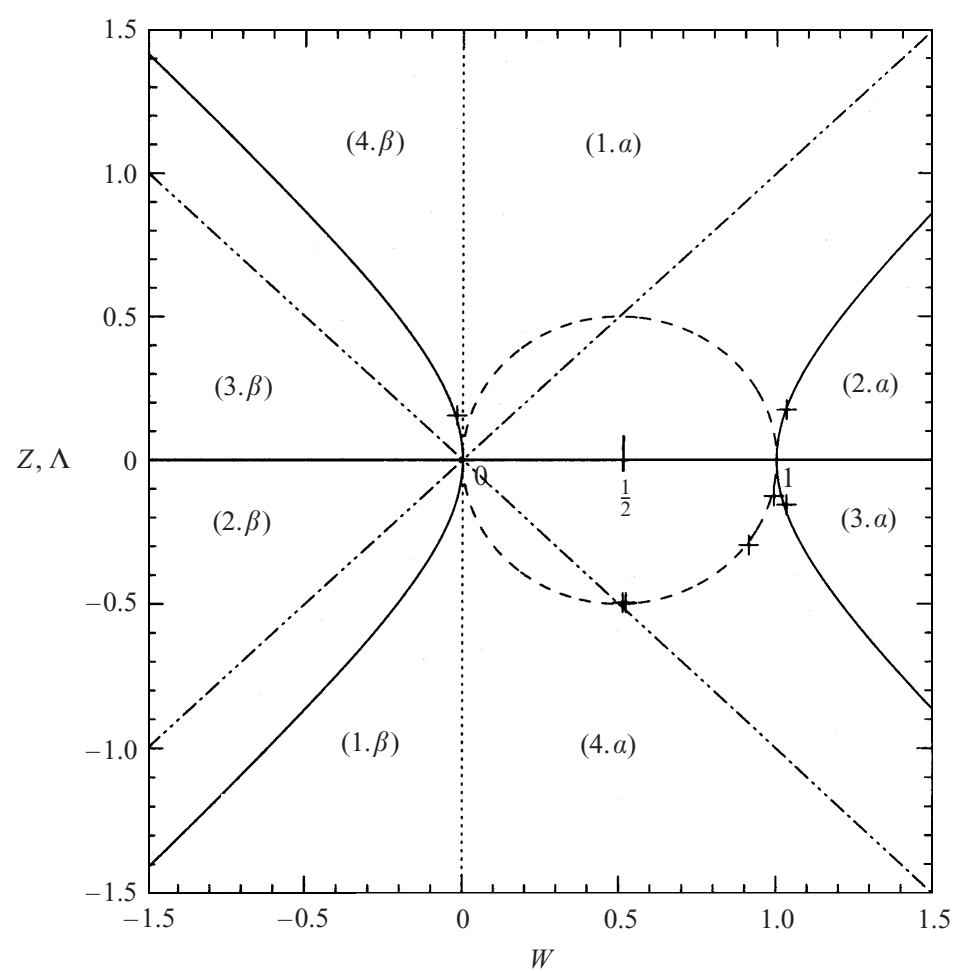

Figure 7. Geometrical representation of the classification of the shock, antishock, hollow and bump defects. The axes and the bisecting lines define eight sectors, which correspond to the configurations introduced in Sec. 3 and are labelled according to the classification of Sec. 3.1. The equilateral hyperbola represents the admissible configurations. We have tried to render the dynamics by the use of different codings: a solid line for the $W$ axis, which is never crossed; a dash-dotted line for the bisecting lines; they are semipermeable frontiers, and are crossed slowly; a dotted line for the $Z$ axis, also a semipermeable frontier, but easily crossed. When possible, the admissible configurations of Table 1 have been represented by crossed. Using the transform $Z=i \Lambda$, the same plane is used to represent the circle of the admissible shock/hollow (or bump) configurations: the appearance of complex residues supplies a connecting path between the two branches of the hyperbola. The four configurations of Table 4 are represented by crosses on the circle.

ations have eventually reached one of the four admissible configurations described in Sec. 2. However, the four admissible configurations do not seem to play an equivalent role in terms of their basin of attraction. At moderate times, six of the initial configurations have been attracted by the two admissible configurations for which $v$ has an antishock and the ratio of magnetic to kinetic energy is larger than one,

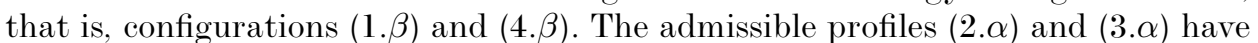
been reached only by initial configurations that were already of the same type. This is in keeping with our previous remark that the fits with the profiles of Sec. 2 were specially good for $(1 . \beta)$ and $(4 . \beta)$.

To pursue this discussion and present the results for large time, we have found it convenient to use a geometrical representation of our classification in eight different configurations. We recall that for the travelling-wave solutions, the (normalized) residues of the (complex) poles are real and related by (2.12); also, the ratio of the residues $D$ is an indication of the relative importance of the magnetic and kinetic 
energies. It is then convenient to consider a plane with coordinates $W$ and $Z$ (see Fig. 7); then (2.12) can be rewritten as

$$
\left(\frac{W-\frac{1}{2}}{\frac{1}{2}}\right)^{2}-\left(\frac{Z}{\frac{1}{2}}\right)^{2}=1,
$$

which shows that the admissible configurations 'live' on an equilateral hyperbola; we note in passing that the origin $(W=Z=0)$ is meaningless while the other apex corresponds to the Burgers equation $(W=1, Z=0)$. The ratio of magnetic to kinetic energy can also be given a geometrical meaning with the aid of the two bisecting lines described by the equation $|D|=|Z / W|=1$. Clearly, the topology of the defect is governed by the position with respect to the main axes, while the energy ratio is governed by the position with respect to the bisecting lines. The plane is thus divided into eight regions corresponding to the eight configurations of our classification, the designations of which are given on Fig. 7. Each of our initial conditions $(3.1 \mathrm{a}, \mathrm{b})$ lies in the corresponding sector of Fig. 7 , but none of them is on the hyperbola, or on the axes, or on the bisecting lines. Using this geometrical language, all our findings concerning the dynamics at times of order one and at moderate times are easily restated:

(i) the basins of attraction of the two sectors $(2 . \alpha)$ and (3. $\alpha)$ seem to be limited to themselves respectively;

(ii) all other points have reached the left half-plane and specifically its sectors $(1 . \beta)$ and $(4 . \beta)$;

(iii) the motion of the representative points always joins points with the same sign of $Z$;

(iv) the slow dynamics of types (2. $\beta$ ) and (3. $\beta$ ) may be linked to the necessity of crossing the bisecting lines, which are the lines of equipartition of energy.

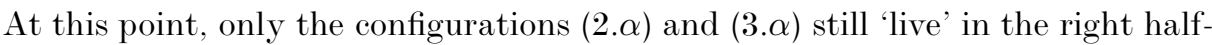
plane; they do have profiles that, at least locally, are well described by those of the meromorphic solutions of Sec. 2 that correspond to the right branch of the hyperbola. Interestingly enough, the study at later times, $O(100)$, revealed that even these configurations would leave the right half-plane and reach a final state of type (4. $\beta$ ) or (1. $\beta$ ) - that is, one of those well described by the meromorphic solutions corresponding to the left branch of the hyperbola. Watching the evolution for even larger times (up to 600), we found that all solutions, whatever their original type, were better and better represented by (2.19) with $|D|$ larger than one. In Fig. 8 , we have given the profiles of the two fields, with the corresponding fits, for two configurations that were initially in two different half-planes and both outside the attractive sectors. They have both reached the attractive sectors, and the fits are valid at moderate distances $((b),(1 . \beta), t=80)$ or even globally for very large times ((a), (4. $\beta), t=570)$. For the latter case, we stress that the value of the fit goes far beyond the core of the defect, which is in this case of small width. The numerical characteristics of the configuration of Fig. 8 are to be found in Table 3 . Although both configurations 'live' on the left branch of the hyperbola, they are distinctly different from the nonlinearity point of view. There is indeed a factor $O(10)$ between the two analyticity-strip widths; at the same time, the ratio of the fields is extremely close to one in the smooth configuration (1. $\beta$ ), which, as already noticed, is the trace of a quasilinear regime. 

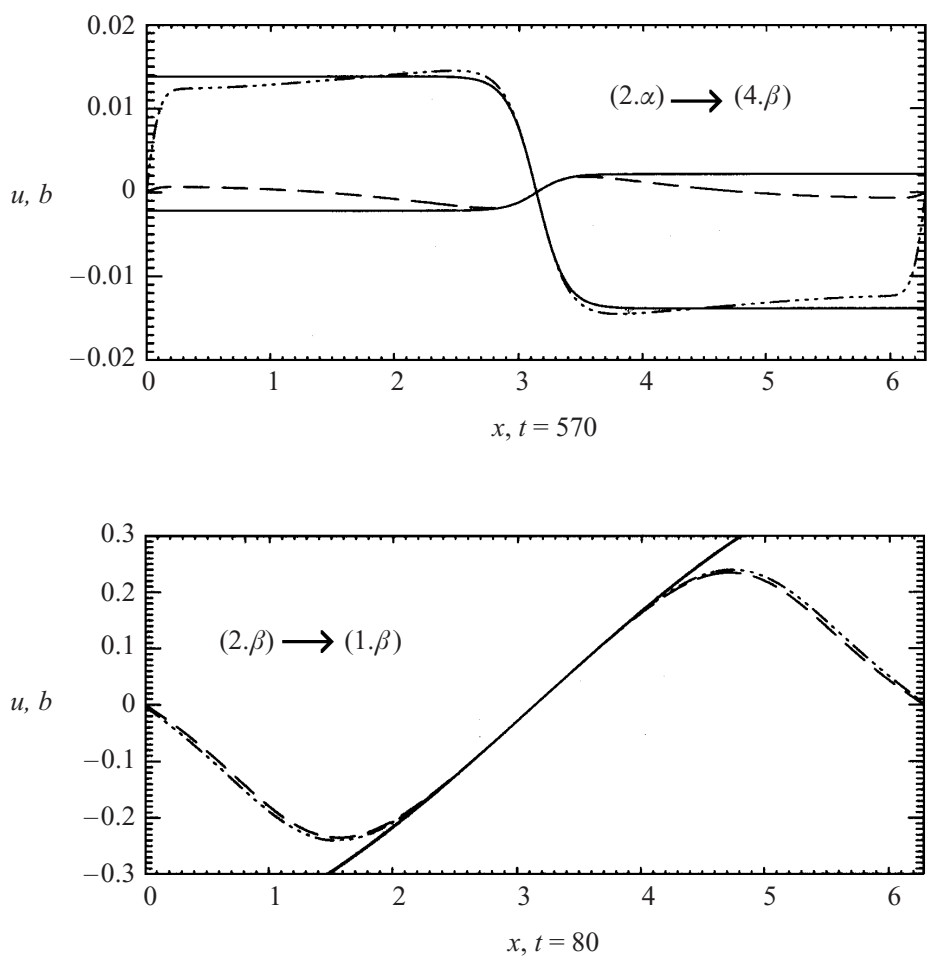

Figure 8. Profiles of the velocity (dash lines) and the magnetic induction (dash-dot lines) for configurations that were initially $(2 . \alpha)$ (a) and $(2 . \beta)(\mathrm{b})$, at times $t=570$ and $t=80$ respectively. Notice the excellent agreement with the fits (solid lines). See Table 3 for numerical details.

Table 3. Numerical values, for times of order hundred, of the ratio $D$, the kinetic residue $W$, the magnetic residue $Z$, the amplitude $\mathscr{A}$, the amplitude $D \mathscr{A}$ and the thickness $\delta$ of the configurations initially $(2 . \alpha)$ and $(2 . \beta)$. See Figure 8 for the profiles and fits.

\begin{tabular}{cccccccl}
\hline$t=0$ & $t=O(100)$ & $D$ & $W$ & $Z$ & $\mathscr{A}$ & $D \mathscr{A}$ & \multicolumn{1}{c}{$\delta$} \\
\hline$(2 . \alpha)$ & $(4 . \beta)$ & -6.36 & -0.025 & 0.161 & 0.002 & -0.014 & 0.366 \\
$(2 . \beta)$ & $(1 . \beta)$ & 1.01 & -84.1 & -84.6 & 0.578 & 0.584 & 4.57 \\
\hline
\end{tabular}

We now try to bring out some general features of the dynamics of the shock defects. Using the geometrical language introduced above, we recall that two sectors were first attracted by the right branch of the hyperbola but that, later on, this region emptied onto the left branch of the hyperbola. Among the admissible configurations, one thus must make the difference between metastable and stable states. Using the parametrization (2.13) of the hyperbola, one sees that the stable branch corresponds to $D \in]-\infty,-1[\cup]+1,+\infty[$; as a result, in those states, (i) the velocity always has the topology of an antishock; (ii) the magnetic energy is larger than the kinetic energy. We also note that the sign of $Z$ seems to be an invariant in any evolution, and so also is the topology of the magnetic induction defect. We suggest that, owing to the lack of dissipation in the magnetic induction equation, the tendency of the system to reach states with a magnetic energy larger than the kinetic one is a governing factor of the dynamics. Combined with the meromorphic 
properties of the travelling waves, which is expressed by the hyperbola, this yields an explanation of all the features described above.

\section{Magnetic hollow defects and complex residues}

We turn now to an other observation made by Passot in the same numerical study alluded to in Sec. 1. Starting with the initial condition

$$
\begin{gathered}
u(x, 0)=\sin x, \\
b(x, 0)=0.2,
\end{gathered}
$$

he found that at times of order one, the Thomas model generated a shock for the velocity field while the magnetic induction had a hollow profile (see Fig. 9, which reproduces our own calculations). We interpret this as an oscillation created by the system to join two separate regions where the magnetic induction has the same (almost) constant value. The position of the bottom of the magnetic hollow coincides with the position of the centre of the velocity shock. Each of the two fields thus exhibits a defect, with the cores at the same place. The combination of the two profiles is thus a new sort of defect naturally generated by the Thomas model. After Passot's discovery, various approximations or interpretations of this phenomenon have been proposed, mainly based on the invariances of the equations. However, all of them implied a type of singularity ruled out by the analyticity properties of (1.1) studied by Passot and Pouquet (1986) and further explored by ourselves (see Secs. 2 and 5). Hereinafter, we propose a simple explanation of the phenomenon based on the meromorphy of the solutions; specifically, we make use of the freedom left by (2.12) to have imaginary residues.

In our 'poor man's model' of a shock (see (3.6)), we considered a meromorphic function with only one pair of poles, no additional entire part, and identical real residues. A more general requirement would be to have complex residues, conjugate of each other. Here we concentrate on the particular case where the residues are purely imaginary. Let us denote by $i \Lambda$ the normalized residue of the pole located at $\pi+i \delta$, with $\delta$ positive; the corresponding rational function thus reads

$$
f(z, t)=(-2 \sigma)(-2 \Lambda) \frac{\delta(t)}{(z-\pi)^{2}+\delta^{2}(t)},
$$

where $\Lambda$ is a priori any real number. The poor man's model of the hollow shape is readily obtained in choosing $\Lambda$ negative. One can imagine describing simultaneously the velocity profile by the corresponding poor man's model of the shock shape. The poles have to be located at the same position as those of the hollow shape, but their (normalized) residues have to be real. Now the crucial remark to be made here is that such a configuration is allowed by the relation (2.12). Assuming indeed that $Z=i \Lambda,(2.12)$ becomes

$$
\Lambda^{2}=W-W^{2}
$$

which has real solutions with $\Lambda \in\left[-\frac{1}{2}, \frac{1}{2}\right]$, provided that $W \in[0,1]$. Prompted by this remark, we have performed a new detailed high-resolution numerical simulation with the initial condition (4.1), with the purpose of providing an explanation of the hollow defect by the meromorphic properties of the system (1.1). On the pattern of the previous section, we have tested this idea in comparing the numerical solutions to the profiles predicted by two different meromorphic models and the numerical 
Fourier data with the asymptotic behaviour of the Fourier transform of the same models. The physical and numerical specifications of the simulations are identical to those of Sec. 3.

Our first model of the magnetic hollow defect is obtained using a slight improvement of the above poor man's model. The latter implies that the field tends to zero at large distances. Figure $9(\mathrm{c})$ clearly shows that the magnetic induction tends to a non-zero constant. We thus just use (3.6) and (4.2), but we allow a background $b_{c}(t)$ for the magnetic induction. Rewriting with our usual notation, we thus have

$$
\begin{gathered}
v(x, t)=(-2 \sigma) \frac{2}{1+\Delta^{2}} \frac{x-\pi}{(x-\pi)^{2}+\delta_{V}^{2}(t)}, \\
b(x, t)=b_{c}(t)+(-2 \sigma) 2 \frac{(-\Delta)}{1+\Delta^{2}} \frac{\delta_{M}(t)}{(x-\pi)^{2}+\delta_{M}^{2}(t)},
\end{gathered}
$$

where $\Delta=Z / i W ; Z$ is an imaginary number. In this model, the Fourier transform of the velocity field is given exactly by (3.7), with $\tilde{A}=1 /\left(1+\Delta^{2}\right)$; the Fourier transform of the magnetic induction can also be calculated exactly, and reads

$$
\hat{b}(k, t)=2 \pi b_{c}(t) \delta_{0}(k)+(-2 \sigma) \frac{(-\Delta)}{1+\Delta^{2}}(2 \pi) e^{-i k \pi} e^{-k \delta_{M}(t)},
$$

where $\delta_{0}(k)$ is the Dirac distribution centred at the origin (we have used Table 2).

We immediately introduce our second model. As in the shock defects, we try to have a better description using degenerate elliptic functions that have an infinite number of poles 'above' the core of the defect. For the velocity field, one uses the usual tanh profile as in the preceding section (see (3.8))

$$
\begin{aligned}
v(x, t) & =-\mathscr{A} \tanh \left(\frac{\pi}{2} \frac{x-\pi}{\delta_{V}(t)}\right), \\
\mathscr{A} & =2 \sigma \frac{1}{1+\Delta^{2}} \frac{\pi}{2} \frac{1}{\delta_{V}(t)} .
\end{aligned}
$$

To build a model for the magnetic induction, we take into account the analyticity constraints coming from (4.7) and (1.1). The analytic extension of the magnetic induction model must have poles located at the same place as the velocity model. To describe a hollow profile, our first model suggests that the residues should be imaginary, with the first pole in the upper complex half-plane $\left(z_{*}=\pi+i \delta_{V}\right)$ having a residue $Z=i \Lambda$, with

$$
\Lambda=\frac{\Delta}{1+\Delta^{2}}
$$

a negative real number. Combining (1.1), written on the imaginary axis, with the ansatz (4.7) on the whole plane, one can prove that $b^{2}$ must be periodic, with period $i \pi$. Moreover, over a distance of the order of the extension $\delta(t)$ of the core of the defect, in any direction in the complex plane, the prediction of the two hollow models should be close to each other. Now, in the simple hollow model (4.2), the function never vanishes except at infinity. Given the pattern of the poles on the imaginary axis and given this non-zero-crossing property, it is unavoidable that the (imaginary) residues have alternating signs. The magnetic induction $b$ is thus periodic of period $2 i \pi$. Forgetting for a moment about a possible additional entire part, the only choice left is, up to a scaling factor on the variable and the field, the degenerate case $(m=1)$ of the Jacobi elliptic function $\operatorname{cn}(z, m)$, that is $(\cosh z)^{-1}$. 

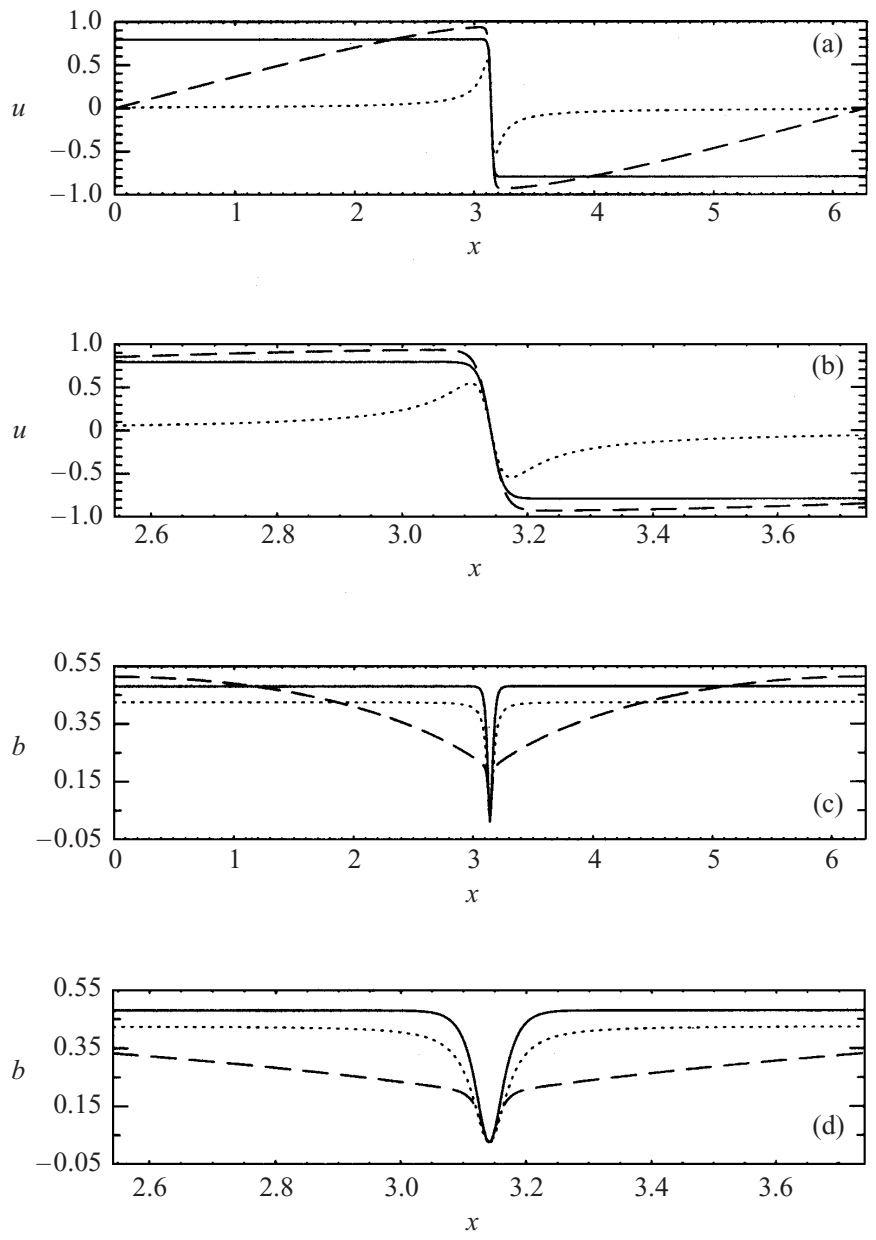

Figure 9. Profiles (dashed lines) of the velocity $(a, b)$ and the magnetic induction $(c, d)$ for time $t=1.6$. Local fits of the profiles realized with the 'poor man's models', (4.4) and (4.5), are shown by dotted lines, and the local fits realized with the elaborate models, (4.7) and (4.9), by solid lines. Note the quality of the fits on the blow-ups (b) and (d). The initial conditions are given by (4.1).

Introducing again a constant background $b_{c}(t)$, and with all our previous notations, our more elaborate model for $b$ thus reads

$$
\begin{gathered}
b(x, t)=b_{c}(t)-\mathscr{B}\left[\cosh \left(\frac{\pi}{2} \frac{x-\pi}{\delta_{M}(t)}\right)\right]^{-1}, \\
\mathscr{B}=-\Delta \mathscr{A}=-2 \sigma \frac{\Delta}{1+\Delta^{2}} \frac{\pi}{2} \frac{1}{\delta_{M}(t)}, \\
\delta_{M}(t)=\delta_{V}(t) .
\end{gathered}
$$

Going to the Fourier space, the transform of the velocity field (4.7) is given exactly by (3.9), with $\tilde{A}=1 /\left(1+\Delta^{2}\right)$; the Fourier transform of the magnetic induction can 


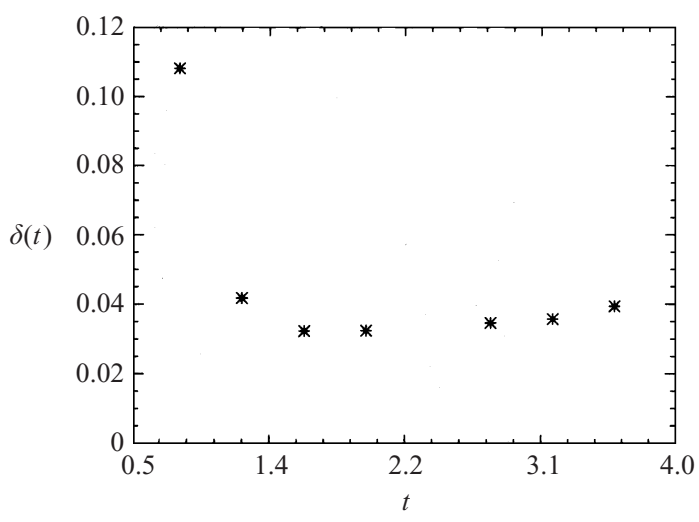

Figure 10. Temporal evolution of the thickness $\delta$ of the shock/hollow defect calculated using the meromorphic 'elaborated' model. The initial conditions are given by formula (4.1).

also be calculated exactly, and reads

$$
\hat{b}(k, t)=2 \pi b_{c}(t) \delta_{0}(k)+(-2 \sigma) \frac{(-\Delta)}{1+\Delta^{2}} \pi e^{-i k \pi}\left\{\cosh \left[k \delta_{M}(t)\right]\right\}^{-1}
$$

(we have used Table 2). Trivial manipulations on (4.10) will again produce, to the dominant order, the regular part of the formula (4.6). Our two meromorphic models of the hollow/shock defect of the Thomas system thus finally both lead to the following predictions concerning the numerical Fourier data:

$$
\begin{gathered}
\text { Phase }[\hat{v}(k, t)]=(-1)^{k} \frac{\pi}{2}, \\
\ln \left[|\hat{v}(k, t)|^{2}\right] \simeq \ln \left(16 \sigma^{2} \pi^{2}\right)-\ln \left[\left(1+\Delta^{2}\right)^{2}\right]-2 \delta_{V}(t) k, \\
\text { Phase }[\hat{b}(k, t)]=\pi \frac{1+(-1)^{k}}{2}, \\
\ln \left[|\hat{b}(k, t)|^{2}\right] \simeq \ln \left(16 \sigma^{2} \pi^{2}\right)-\ln \left[\left(\frac{1+\Delta^{2}}{\Delta}\right)^{2}\right]-2 \delta_{M}(t) k,
\end{gathered}
$$

for

$$
k \gg 1 \text {. }
$$

Using the numerical data provided by our new simulation mentioned above, we have tested the predictions of our two models in both physical and Fourier space. With regard to the Fourier space, we have concentrated on the predictions concerning the phase, for which the presence of an imaginary residue is a key factor (compare (4.13) with (3.12)). In the shock/hollow defect, we found agreement up to the 11th digit between the Fourier predictions $(4.11),(4.14)$ and the numerics. For the physical space, we have tried to fit the numerical profiles by the analytic expressions of the first and second models. We proceed as follows. From the expressions (4.4), (4.5) and (4.7), (4.9), it is an easy matter to obtain predictions for the infima of the gradients of both fields, and thus for the ratio of these quantities. Hence, by comparison with the numerical data, one can find the value of $\Delta$, the ratio of the amplitude of the velocity shock to the depth of the magnetic hollow. From the value of the infimum of the gradient of the velocity field, one then gets a 
Table 4. Numerical values, for times of order one, of the ratio $\Delta$, the kinetic residue $W$, the magnetic residue $\Lambda$, the amplitude $\mathscr{A}$, the amplitude $|\Delta \mathscr{A}|$, the background $b_{c}$ and the thickness $\delta$ of four different shock/hollow defects. As the initial magnetic induction $\mathscr{B}_{0}$ increases, the amplitude of the velocity shock $\mathscr{A}$ decreases.

\begin{tabular}{rlrrrr}
\hline \multirow{2}{*}{$t=0$} & $\mathscr{U}_{0}$ & 1 & \multicolumn{1}{c}{1} & \multicolumn{1}{c}{1} & \multicolumn{1}{c}{1} \\
& $\mathscr{B}_{0}$ & \multicolumn{1}{c}{0.1} & \multicolumn{1}{c}{0.2} & \multicolumn{1}{c}{0.5} & \multicolumn{1}{c}{1.5} \\
\hline & $\Delta$ & -0.185 & -0.574 & -1.055 & -1.078 \\
$W$ & 0.967 & 0.752 & 0.473 & 0.462 \\
$\Lambda$ & -0.178 & -0.432 & -0.499 & -0.499 \\
& $\mathscr{A}$ & 0.950 & 0.793 & 0.401 & 0.110 \\
& $|\Delta \mathscr{A}|$ & 0.176 & 0.455 & 0.423 & 0.119 \\
$b_{c}$ & 0.1 & 0.2 & 0.5 & 1.5 \\
& $\delta$ & 0.032 & 0.030 & 0.037 & 0.132 \\
\hline
\end{tabular}

numerical estimate of the thickness $\delta(t)$ or equivalently of the amplitude $\mathscr{A}$ of the shock. Depending on the model, $\mathscr{A}$ and $\delta$ are related by

$$
\delta=\frac{2 \sigma}{\mathscr{A}\left(1+\Delta^{2}\right)}
$$

in the poor man's model, and

$$
\delta=\frac{\pi}{2} \frac{2 \sigma}{\mathscr{A}\left(1+\Delta^{2}\right)}
$$

in the elaborated model. Finally, using the expression for the magnetic field at the centre $x=\pi$, one adjusts the numerical value of the magnetic background. The output of this process is summarized in Figs 9 and 10 and in Table 4. In Fig. 9(a), the numerical profile of the velocity is plotted along with the fits provided by our two models; Fig. $9(\mathrm{~b})$ is a blow-up centred at the core of the defect. The same pattern is followed for the magnetic induction in Figs $9(\mathrm{c}, \mathrm{d})$. Both models provide a good description of the core of the defect. As could be expected, the more elaborate model has a more global validity; moreover, the resulting fits agree remarkably well with the numerical solution near the centre of the magnetic hollow defect. In Fig. 10, we present the evolution of the thickness of the defect, calculated using the elaborated model and (4.17), at times of order one. As in Fig. 4, one can distinguish a nonlinear regime that produces the defect and a dissipative regime where the spatial extension of the defect increases.

Finally, for a more complete exploration, we have performed numerical experiments with three other initial conditions of the type (4.1), that is, with a uniform initial magnetic induction. Together with the simulation discussed above at length, we have a set of four experiments with initial condition

$$
\begin{gathered}
u(x, 0)=\sin x, \\
b(x, 0) \equiv b_{0},
\end{gathered}
$$

with $b_{0}=0.1,0.2,0.5$ and 1.5. At times of order one, the four have evolved towards a shock/hollow defect, for which we have performed a fit of the profiles, using the elaborated model (this time we have put aside the possibility of negative $b_{0}$, which of course would produce shock/bump defects). The corresponding parameters are presented in Table 4 . One notes that, as $b_{0}$ increases, the velocity residue $W$ decreases and the width $\delta$ increases; accordingly the velocity shock amplitude $\mathscr{A}$ decreases. The system is thus less and less nonlinear, the effect being sharp between 
the third and fourth experiments. Again, this can be interpreted in terms of the influence of the asymptotic regime $\sigma=\eta=0$. Indeed, in the work already referred to in Sec. 3, Sulem et al. (1979) proved that in this regime and with regular initial conditions, regularity in $\mathbb{R}$ is preserved at all times for the Thomas system, under the condition $\inf |b(x, 0)|>\frac{1}{2}[\sup u(x, 0)-\inf u(x, 0)]$. This is achieved only with the fourth initial condition, for which a sufficiently strong initial magnetic induction field smoothes out the nonlinear effects.

To conclude, we go back to the geometrical interpretation of the relation between the (normalized) residues of the velocity and of the magnetic induction. To describe the shock/hollow defect, we have been led to assume real residues for the velocity field and purely imaginary residues for the magnetic induction. With the notation $Z=i \Lambda$ already introduced, (3.15) becomes

$$
\left(\frac{W-\frac{1}{2}}{\frac{1}{2}}\right)^{2}+\left(\frac{\Lambda}{\frac{1}{2}}\right)^{2}=1,
$$

which defines the circle of the admissible hollow (bump) configurations in the $(W, \Lambda)$ plane. In Fig. 7, we have mapped this plane onto the real $(W, Z)$ plane to draw this circle. The outcome of our four numerical experiments appears as crosses located on this circle; as $b_{0}$ increases, the crosses travel in the $(W, \Lambda)$ plane down the lower right arch. The circle fills the gap between the two branches of the hyperbola, showing that real values of the velocity field residues $W \in[0,1]$ are allowed, and even have a very interesting physical interpretation.

\section{Partial integrability}

The Thomas system (1.1) is not integrable, even with the restriction that $\eta$ equals zero. Nevertheless, the stationary subcase has been integrated in Sec. 2. Passot and Pouquet (1986) noted that a certain self-similar reduction of (1.1) is meromorphic in the reduced variable. Moreover the full system $(1.1)(\eta=0)$ exhibits properties and symmetries that are usually linked to integrability. We present here some of these features, thus proving that the Thomas system with zero magnetic resistivity is what is now called a partially integrable system (see below).

Our discussion relies mainly on the singularity analysis of the system, with both a local and global approach. Historically, since the days of Kovaleskaya and Painlevé, integrability of ordinary differential systems is associated with meromorphy of the integrals of the system. The modern point of view is that multivalued (even infinitely so) singularities are acceptable (e.g. rational branch points, logarithms or complex exponents) provided they are not 'too numerous'. Another step was taken when the Painlevé-type singularity study was extended to PDE's (Weiss et al. 1983). The idea here is to build a sort of functional Painlevé-Laurent expansion of the solution around a manifold that contains all its singularities. In both cases (ODEs and PDEs), the expansion, if performed for the general solution of a nonintegrable case, will either break down or diverge or depend on a number of free parameters smaller than the order of the system. In the latter case, the general solution clearly escapes to the singularity analysis but the expansion may represent a subfamily of, for example, meromorphic exact solutions. One can then speak of partial integrability. 


\subsection{The Painlevé-WTC expansion}

The goal is to extend and generalize as much as possible the simple leading-order analysis presented in Sec. 2.2. The main object is the singularity manifold $\Phi(x, t)$; the zeros of the function $\Phi(x, t)$, obtained for real or complex $x$ and $t$, are the singularities of the function under study. It is assumed that $\Phi$ has an analyticity domain containing all these zeros. The structure of the system (1.1) implies that any singularity for the velocity field will be accompanied by a singularity for the magnetic induction with the same spatio-temporal location. As a result, the singularity manifold of $v$ is identical to its counterpart for $b$. Using a leading-order analysis, one is easily convinced that the solutions of our system are proportional to $\Phi^{-1}$ near the zeros of $\Phi$. We thus assume the following functional form:

$$
\begin{aligned}
& v=\frac{1}{\Phi} \sum_{m \geqslant 0} v_{m} \Phi^{m}, \\
& b=\frac{1}{\Phi} \sum_{m \geqslant 0} b_{m} \Phi^{m},
\end{aligned}
$$

for respectively the velocity and the magnetic induction field. Here the $v_{m}$ and the $b_{m}$ are functions of $x$ and $t$ with analyticity properties in the same domain as $\Phi$. The next step is to use these representations in the system (1.1) with $\eta=0$. This will produce conditions on $v_{0}$ and $b_{0}$ and recurrence relations for the subsequent $v_{m}$ and $b_{m}$. The analysis here is thus an asymptotic one, where the ordering is governed by the successive integer powers of $\Phi$. We envisage later the possibility of logarithmic corrections, but we never attack the formidable problem of the summability of the complete sequence of asymptotic terms. Thus we concentrate on the discussion of the formal existence of the recurrence scheme, which we consider now.

Balancing the two leading order terms $\left(O\left(\Phi^{-3}\right)\right)$ in $(1.1 b)$ with $\eta=0$ yields

$$
\left(-\Phi_{x}+\Phi_{x}\right) v_{0} b_{0}=0
$$

thus leaving at this point $v_{0}$ and $b_{0}$ entirely free. The mechanism is that the multiplicative factor of $v_{0} b_{0}$ vanishes, a circumstance called a 'resonance', and at the same time the inhomogeneous term also vanishes, making the resonance a 'compatible' one. The free functions are thus at our disposal, playing the role of integrals of the motion in the integration of a usual differential system. Turning now to the first equation (1.1a) of the Thomas system, and balancing the $O\left(\Phi^{-3}\right)$ terms (i.e. two nonlinear terms and the dissipative term), we get

$$
v_{0}^{2}-b_{0}^{2}+2 \sigma \phi_{x} v_{0}=0 .
$$

Here a restriction appears, $b_{0}$ and $v_{0}$ being linked by an algebraic equation. The situation thus lies between the Burgers case, where the residue function is entirely determined by the algebraic structure of the dynamical equation $\left((5.3)\right.$ with $\left.b_{0} \equiv 0\right)$, and a fully fledged compatible resonance, where $v_{0}$ and $b_{0}$ would both be entirely free. We see that the resonance is incomplete. We further note that the second order of the recursion scheme will induce some feedback constraint on these first functions. In keeping with the idea of an easy comparison with the pure Burgers case, we introduce normalized amplitudes $P$ and $R$, such that

$$
\begin{aligned}
& v_{0}=-2 \sigma P, \\
& b_{0}=-2 \sigma R .
\end{aligned}
$$


The leading-order relation (5.3) then reads

$$
R^{2}=P^{2}-P \Phi_{x},
$$

which from now on we shall call the 'singularity relation' (SR). By a further transform, we can introduce the normalized residues $W(x, t)$ and $Z(x, t)$ such that

$$
\begin{aligned}
& P=W \Phi_{x}, \\
& R=Z \Phi_{x} ;
\end{aligned}
$$

the SR then reads

$$
Z^{2}=W^{2}-W,
$$

a familiar form from the travelling-wave study, but now with space- and timedependent residues.

Balancing in (1.1) $(\eta=0)$ the terms of next to leading order, and so on, will produce at each step $m \geqslant 1$ a set of algebraic (i.e. not differential) linear inhomogeneous equations for the pair $\left\{v_{m}, b_{m}\right\}$. The coefficients of $v_{m}$ and $b_{m}$ depend on $\{\Phi, P, R\}$; the inhomogeneous term depends on the intermediate asymptotic terms $\left\{v_{n}, b_{n} ; 0 \leqslant n<m\right\}$ and their derivatives-hence the recurrence structure of the scheme.

The first set is obtained in balancing terms $O\left(\Phi^{-2}\right)$ in (1.1); it reads

$$
\begin{gathered}
\Phi_{x} v_{1}=-\Phi_{t}+\sigma\left(2 P_{x}-\frac{\Phi_{x x}}{\Phi_{x}} P\right), \\
R \Phi_{x} b_{1}=\sigma P\left(2 P_{x}-\frac{\Phi_{x x}}{\Phi_{x}} P\right)-\sigma P_{x} \Phi_{x} .
\end{gathered}
$$

Using the $\mathrm{SR},(5.8 \mathrm{~b})$ can be rewritten as

$$
\Phi_{x} b_{1}=\sigma\left(2 R_{x}-\frac{\Phi_{x x}}{\Phi_{x}} R\right),
$$

a form more symmetric to (5.8a).

The following step $(m=2)$ has a manifold outcome that is crucial for the present discussion. Balancing the $O\left(\Phi^{-1}\right)$ terms in (1.1) yields

$$
\begin{gathered}
0=-P_{t}-\left(P v_{1}-R b_{1}\right)_{x}+\sigma P_{x x}, \\
\Phi_{x}\left(R v_{2}-P b_{2}\right)=\frac{1}{2}\left[R_{t}-\left(R v_{1 x}-R_{x} v_{1}\right)+\left(P b_{1 x}-P_{x} b_{1}\right)\right] .
\end{gathered}
$$

Clearly we again have an incomplete resonance. Only in the first equation does the left-hand side vanish. The right-hand side must vanish simultaneously to have a consistent result and a compatible resonance. Since the right-hand side depends on the previous functional coefficients, it appears as a back-constraint on $P, R, v_{1}$ and $b_{1}$, as announced above; using (5.8), (5.9a) can indeed be rewritten as

$$
P_{t}=\left(P \frac{\Phi_{t}}{\Phi_{x}}\right)_{x} ;
$$

(from (5.8) and the SR, an identical relation can be written for $R$ ). We comment that, even at the simple formal level at which we are staying here, the whole recurrence scheme cannot exist if (5.10) is not satisfied by $P$. If we are to hope for explicit meromorphic solutions of the Thomas system, the very existence of their Painlevé-Laurent expansion implies (5.10), which we thus from now on call the 
'integrability restriction' (IR). We note in passing that, using (5.6), the IR may be transformed into

$$
W_{x} \Phi_{t}=W_{t} \Phi_{x}
$$

Considering $\Phi$ as given, this is a first-order linear PDE where the unknown $W$ appears only through its first-order derivatives; it can easily be reduced to

$$
W(x, t)=W\{\Phi(x, t)\}
$$

that is, $W$ depends on $x$ and $t$ only through $\Phi(x, t)$ or, in other words, it is a function of $\Phi$ alone. This is a restriction on (5.7): not only are $Z$ and $W$ algebraically related, but in addition $W$ cannot be any given function. Up to this point, $v_{2}$ and $b_{2}$ are free. However, the second equation $(5.9 \mathrm{~b})$ exhibits a constraint on a certain linear combination of $v_{2}$ and $b_{2}$. The freedom that is left corresponds to a one-dimensional vector subspace instead of a two-dimensional space. If one wants more freedom in the choice of $\left\{v_{2} ; b_{2}\right\}$, it will be at the cost of restrictions on the previous terms (see the right-hand side of $(5.9 \mathrm{~b})$ ); the consequences will play a role in Secs 5.2 and 5.3. We comment here that the imperfect character of the resonances at $m=0$ and $m=2$, is the ground for saying 'partial integrability'.

The third step of the recurrence yields the two following equations for $v_{3}$ and $b_{3}$ :

$$
\begin{aligned}
2 \sigma \Phi_{x}[(P & \left.\left.-3 R+\Phi_{x}\right) v_{3}+(3 P-R) b_{3}\right]=T_{v}\left\{v_{1} ; b_{1}\right\}+T_{b}\left\{v_{1} ; b_{1}\right\} \\
& +v_{2}\left[\Phi_{t}-2 \sigma\left(P_{x}+R_{x}\right)+\Phi_{x}\left(v_{1}-b_{1}\right)-\sigma \Phi_{x x}\right] \\
& +b_{2}\left[\Phi_{t}+2 \sigma\left(P_{x}+R_{x}\right)+\Phi_{x}\left(v_{1}-b_{1}\right)\right] \\
& -2 \sigma v_{2 x}\left(P-R+\Phi_{x}\right)-2 \sigma b_{2 x}(P-R), \\
2 \sigma \Phi_{x}[- & \left.\left.P+3 R+\Phi_{x}\right) v_{3}+(3 P+R) b_{3}\right]=-T_{v}\left\{v_{1} ; b_{1}\right\}+T_{b}\left\{v_{1} ; b_{1}\right\} \\
& -v_{2}\left[\Phi_{t}-2 \sigma\left(P_{x}-R_{x}\right)+\Phi_{x}\left(v_{1}+b_{1}\right)-\sigma \Phi_{x x}\right] \\
& +b_{2}\left[\Phi_{t}+2 \sigma\left(P_{x}-R_{x}\right)+\Phi_{x}\left(v_{1}+b_{1}\right)\right] \\
& +2 \sigma v_{2 x}\left(P+R+\Phi_{x}\right)-2 \sigma b_{2 x}(P+R),
\end{aligned}
$$

where

$$
\begin{aligned}
& T_{v}\left\{v_{1} ; b_{1}\right\}=v_{1 t}+v_{1} v_{1 x}-b_{1} b_{1 x}-\sigma v_{1 x x}, \\
& T_{b}\left\{v_{1} ; b_{1}\right\}=b_{1 t}+v_{1} b_{1 x}-b_{1} v_{1 x} .
\end{aligned}
$$

The relations (5.13) and (5.14) will be useful later; this is why we have singled them out, along with the fundamental outputs of the asymptotic analysis at order $\Phi^{-3}(m=0), \Phi^{-2}(m=1)$ and $\Phi^{-1}(m=2)$. It should be realized, however, that from order $m=2$ onwards, a general formula can be given, valid at any order $m \geqslant 2$ of the recurrence, namely

$$
\sigma \Phi_{x} \mathbf{M}_{m}\left(\begin{array}{c}
v_{m} \\
b_{m}
\end{array}\right)=\left(\begin{array}{c}
\mathscr{F}^{+} \\
\mathscr{F}_{m} \\
m
\end{array}\right)
$$

where $\mathbf{M}_{m}$ is the matrix

$$
\mathbf{M}_{m}=\left(\begin{array}{cc}
2 m R+2(m-2) P+\Phi_{x}(m-2)(m-1) & -2 m P-2(m-2) R \\
2(m-2) P-2 m R+\Phi_{x}(m-2)(m-1) & 2 m P-2(m-2) R
\end{array}\right)
$$


and

$$
\begin{aligned}
\mathscr{F}_{m}^{+}= & \left(v_{m-2}-b_{m-2}\right)_{t}+(m-2) \Phi_{t}\left(v_{m-1}-b_{m-1}\right) \\
& +\sum_{l=0}^{m-1}\left(v_{l}+b_{l}\right)\left(v_{m-l-1}-b_{m-l-1}\right)_{x} \\
& +\Phi_{x} \sum_{l=1}^{m-1}\left(v_{l}+b_{l}\right)\left(v_{m-l}-b_{m-l}\right)(m-l-1) \\
& -\sigma\left[\left(v_{m-2}\right)_{x x}+(m-2) \Phi_{x}\left(2 v_{m-1}\right)_{x}+(m-2) \Phi_{x x} v_{m-1}\right] \\
\mathscr{F}_{m}^{-}= & \left(v_{m-2}+b_{m-2}\right)_{t}+(m-2) \Phi_{t}\left(v_{m-1}+b_{m-1}\right) \\
+ & \sum_{l=0}^{m-1}\left(v_{l}-b_{l}\right)\left(v_{m-l-1}+b_{m-l-1}\right)_{x} \\
+ & \Phi_{x} \sum_{l=1}^{m-1}\left(v_{l}-b_{l}\right)\left(v_{m-l}+b_{m-l}\right)(m-l-1) \\
- & \sigma\left[\left(v_{m-2}\right)_{x x}+(m-2) \Phi_{x}\left(2 v_{m-1}\right)_{x}+(m-2) \Phi_{x x} v_{m-1}\right] .
\end{aligned}
$$

Using the SR, the determinant $\Gamma_{m}$ of the matrix $\mathbf{M}_{m}$ is readily obtained, and reads

$$
\Gamma_{m}=-4 \sigma^{2} \Phi_{x}^{3} \operatorname{Pm}(m-2)(m+1) .
$$

The resonance at $m=2$ (and, at a formal level, the resonance at $m=0$ ) can be read off from (5.16) as the condition for the vanishing of the determinant of the linear system (5.15); but, at the same time, one sees their incomplete character, since, as is easily checked, the matrix $\mathbf{M}_{m}$ itself never vanishes.

Finally we mention that a rapid exploration suggests that, as in many nonintegrable systems, the introduction of logarithmic corrections may restore the incomplete resonances to completeness (see e.g. Bender and Orszag 1978). In this exploration, one writes $v(x, t)$ and $b(x, t)$ as double series, called $\Psi$ series, here of the form

$$
\begin{aligned}
& v=\frac{1}{\Phi} \sum v_{m, k} \Phi^{m}(\ln \Phi)^{k}, \\
& b=\frac{1}{\Phi} \sum b_{m, k} \Phi^{m}(\ln \Phi)^{k}
\end{aligned}
$$

one finds that (5.3) disappears and is replaced by a double condition on $b_{0,1}, v_{0,1}$ and $v_{0,2}$, the functional coefficients of the first logarithmic corrections. In such a formalism, one has, in general, at hand a sufficient number of free integrals of the motion, but a closed formula for the solution cannot be obtained, given the infinite number of logarithmic corrections. For mathematical purposes, this may be useful (Fournier et al. 1988); for practical purposes, it is too high a cost for forcing integrability in a non- (or partially non-) integrable system. However, it should be remembered that solutions of the type studied, for example in Sec. 4, which are well described assuming simple pure poles as singularities, may actually possess logarithmic corrections. In this sense, the rational and elliptic representations may well be more than simply models, as we called them, but rather low-order expansions resulting from partial resummation of the 'without-log terms' in (5.17).

At this point, we acknowledge our intellectual debt to Passot and Pouquet. In 
their work referred to in Sec. 1, they performed the Painlevé test on the full system (1.1), but they developed the formalism using the favourite Elsässer variables $z^{ \pm}=$ $v \pm b$; they found that the general case is non-integrable, and, for $\eta=0$, they pointed out a condition equivalent to (5.10) in their formalism; however, they were misled by this formalism as to the role of the limiting pure Burgers case and they did not envisage the truncations of the series (5.1), which will be the topic of the next two subsections.

\subsection{The complete truncation: a poor $\tau$ function?}

Suppose $v_{1}=b_{1}=v_{2}=b_{2}=\ldots=v_{m-1}=b_{m-1}=0$, where $m \geqslant 3$; then, by (5.15), $v_{m}=b_{m}=0$. One can thus prove by recurrence that, if $v_{1}=b_{1}=v_{2}=b_{2}=0$, all terms of the series (5.1) vanish except for the lowest one; that is, one has the identities

$$
\begin{aligned}
& v=-2 \sigma \frac{P}{\Phi}, \\
& b=-2 \sigma \frac{R}{\Phi} ;
\end{aligned}
$$

they express the nonlinear transform between the potential $\Phi$ and the physical fields $v$ and $b$; they are reminiscent of the logarithmic Hopf-Cole transform

$$
v=-2 \sigma \frac{\Phi_{x}}{\Phi}
$$

which maps the Burgers equation into a linear problem, and of similar transforms appearing in the calculation of multisoliton solutions to various integrable equations; in the latter context, the nonlinear potential $\Phi$ is called a $\tau$ function (Newell 1985). The difference is that the residues are only partially constrained by the SR. Other differences will appear next.

The cost of this extreme truncation is that the right-hand side of (5.8) and (5.9) vanish altogether. However, the two groups of conditions have a very different status. Coupled with the SR, the conditions coming from (5.8) may be viewed as the dynamical equations of the problem; they play the role of the heat equation in the Burgers case, or of the Hirota bilinear equation for the $\tau$ function in, for example, the Korteveg-de Vries case. They form a system of three equations for three unknown quantities, from which one could expect to derive families of meromorphic solutions, depending on the initial and boundary conditions. But the second group of conditions, obtained as compatibility conditions for an incomplete resonance, adds severe restrictions. In particular, under the present hypothesis, $(5.9 \mathrm{~b})$ reads

$$
R_{t}=0,
$$

which, as we shall see, drastically reduces the number of solutions amenable to this scheme. We now derive explicitly those solutions.

We put aside the completely trivial solution $R=P=0$, which implies $v=b=0$. We next consider the case

$$
\begin{gathered}
R=0, \\
P=\Phi_{x} .
\end{gathered}
$$

Then (5.8a) becomes

$$
\Phi_{t}=\sigma \Phi_{x x}
$$


and (5.9) is satisfied identically; at the same time, (5.18a) reduces to the Hopf-Cole transform (5.19) and (5.18b) to $b=0$. This particular family of solutions is merely the set of all solutions of the Burgers equation. We suppose now that neither $P$ nor $R$ are degenerate, but we make the assumption

$$
\Phi_{t}=0
$$

Combining (5.8a) and (5.8b) yields $P_{x}=0$ (we disregard the case $\Phi_{x}=0$, where the singularity analysis is invalid). Then, from (5.8a), $\Phi_{x x}=0$; and then from (5.8b), $R_{x}=0$. And, from (5.9) we have $P_{t}=R_{t}=0$. Thus, finally,

$$
\Phi=\Gamma\left(x-x_{*}\right),
$$

where $x_{*}$ and $\Gamma$ are pure real constants, with $\Gamma \neq 0$, while $P$ and $R$ are also pure non-zero real constants. Using the normalized residues, we have $P=\Gamma W$ and $R=\Gamma Z$, where $Z$ and $W$ are pure non-zero real constants, related by $Z^{2}=W^{2}-W$. Returning to the physical fields, we have, with the particular assumption $\Phi_{t}=0$, the unique solution

$$
\begin{aligned}
& v=-2 \sigma \frac{W}{x-x_{*}}, \\
& b=-2 \sigma \frac{Z}{x-x_{*}},
\end{aligned}
$$

which is merely the single-pole stationary solution (2.5).

Now the partially integrable character of the Thomas model appears here in the fact that no solution other than Burgers and (5.25) is obtained via the present $\tau$ function ansatz. The proof is left to an appendix, but let it be said here again that the compatibility conditions of the quasiresonance at $m=2$ play a determining role.

\subsection{A Bäcklund transform}

We consider here a less severe truncation of the Painlevé expansion. The technical remark is that one does not need vanishing $v_{1}$ and $b_{1}$ to obtain the proof by recurrence considered at the beginning of the previous subsection. A look at (5.13) will show that $v_{2}=b_{2}=0$ combined with $T_{v}\left\{v_{1} ; b_{1}\right\}=T_{b}\left\{v_{1} ; b_{1}\right\}=0$ will also allow one to crank the machine. This ensures the possibility of a two-term truncation

$$
\begin{aligned}
& v=-2 \sigma \frac{P}{\Phi}+v_{1}, \\
& b=-2 \sigma \frac{R}{\Phi}+b_{1},
\end{aligned}
$$

provided that $v_{1}$ and $b_{1}$ themselves form a solution of the Thomas system with $\eta=0$ (see (5.14)). A more precise statement is that, given the relation (5.26) and under the conditions that the SR, (5.8) and (5.9) with $v_{2}=b_{2}=0$ hold, then $\left\{v_{1} ; b_{1}\right\}$ is a solution of the Thomas system' implies ' $\{v ; b\}$ is a solution of the Thomas system' and conversely. The relation (5.26) thus reveals a sort of nonlinear additivity among the solutions of the Thomas system; given a seed, one can expect that repeated application of (5.26) will produce a family of new solutions of the same Thomas system; in such a situation, one speaks of an auto-Bäcklund transform. We note that the meromorphy assumption for the fields and the potentials is consistent with the transform, and that, in general, the existence of a Bäcklund transform 
is thought of as a favourable symptom for integrability. We also stress that the transform has an inverse; that is,

$$
\begin{aligned}
& v_{1}=-2 \sigma \frac{\tilde{P}}{\tilde{\Phi}}+v, \\
& b_{1}=-2 \sigma \frac{\tilde{R}}{\tilde{\Phi}}+b,
\end{aligned}
$$

provided that

$$
\begin{gathered}
\tilde{P}=-P \frac{L}{\Phi^{2}}, \\
\tilde{R}=-R \frac{L}{\Phi^{2}}, \\
\tilde{\Phi}=\frac{L}{\Phi},
\end{gathered}
$$

where $L$ is a non-zero real pure constant.

A first subclass of solutions satisfying the Bäcklund conjugation is readily obtained on considering the limiting Burgers case. One assumes that $b=b_{1}=0$, and the Thomas-Bäcklund transform then reads

$$
\begin{gathered}
v=-2 \sigma \frac{P}{\Phi}+v_{1}, \\
R=0 ;
\end{gathered}
$$

the SR then implies that $P=\Phi_{x}$; as a consequence, (5.8a) yields

$$
\Phi_{t}+v_{1} \Phi_{x}=\sigma \Phi_{x x},
$$

and $(5.9 \mathrm{a})$ is satisfied identically. The transform (5.29a) and the linear condition (5.30) form the known Bäcklund transform of the Burgers equation, which is recovered here as a limiting case.

In the above derivation, the nonlinear condition (5.8a) becomes linear and the conditions $(5.8 \mathrm{c})$ and $(5.9 \mathrm{a}, \mathrm{b})$ do not play any role. From Sec. 5.2, we know that the conditions (5.9) may impose drastic limitations to the integrability-related features of the problem. To check if our finding of a Bäcklund transform is not deceptive, we have looked for explicit couples $\left\{v_{1} ; b_{1}\right\} /\{v ; b\}$ other than the Burgers one, using various non-trivial ansätze.

The first idea is to hunt for cases where $\Phi_{t}=0$. Through the SR, it can immediately be concluded that $P_{t}=R_{t}=0$. Through (5.8), one obtains also $v_{1 t}=b_{1 t}=0$. One is thus in the realm of stationary solutions, for both $\left\{v_{1} ; b_{1}\right\}$ and $\{v ; b\}$. A detailed examination shows that there is no new admissible set if $v_{1}=0$, or if $\left|v_{1}\right|=\left|b_{1}\right|$ or if $b_{1}=0$. One can thus write $b_{1}=D_{1} v_{1}$, where $D_{1}$ is a pure constant, with $D_{1} \in \overline{\mathbb{R}} \backslash\{0 ; \pm 1 ; \pm \infty\}$. To explore a specific direction, we further assume that $v_{1}$ is a pure, non-zero, real constant. Then (5.9b) implies that $R=D_{1} P+E$, where $E$ is a pure real constant. Combining this information with (5.8) leads to the conclusion that either $\Phi_{x x}$ or $E$ vanishes. The use of the SR shows that the first route is a dead end. Following the second route, and using the SR and (5.8a), one obtains

$$
\begin{gathered}
\Phi_{x}=\left(1-D_{1}^{2}\right) P, \\
P_{x}=\frac{1}{\sigma}\left(1-D_{1}^{2}\right) v_{1} P .
\end{gathered}
$$


Thus, finally

$$
\begin{gathered}
P=\alpha \exp \left[\frac{1}{\sigma}\left(1-D_{1}^{2}\right) v_{1} x\right], \\
\Phi=\frac{\sigma}{v_{1}} P+\beta, \\
R=D_{1} P,
\end{gathered}
$$

where $\alpha$ and $\beta$ are pure real constants with $\alpha \neq 0$. Three different targets may be build with these formulae. First, if $\beta=0$, then $v=-v_{1}$ and $b=-b_{1}$. Then, if $\beta \neq 0$, two different formulae for $v$ are obtained, depending on the sign of $\beta / \alpha v_{1}$. With the positive sign, the final formulae read

$$
\begin{gathered}
v=-v_{1} \tanh \left[\frac{1}{2} \gamma\left(x-x_{*}\right)\right], \\
b=D_{1} v,
\end{gathered}
$$

where

$$
\gamma=\frac{1}{\sigma}\left(1-D_{1}^{2}\right) v_{1}
$$

and $x_{*}$ is a free real number. With the negative sign, the final formulae read

$$
\begin{gathered}
v=-v_{1} \operatorname{coth}\left[\frac{1}{2} \gamma\left(x-x_{*}\right)\right], \\
b=D_{1} v .
\end{gathered}
$$

Starting from uniform fields, the Bäcklund transform thus builds the most interesting stationary solutions (2.8) of Sec. 2. We note as a curiosity that, since the transform may be inverted (see above (5.28)), one can make the easy prediction that the image of the double tanh profile through the Bäcklund transform is a pair of uniform fields.

The second idea is to hunt for cases where the potentials $\Phi, R$ and $P$ have a simple spatial structure. Specifically, we have considered

$$
\begin{gathered}
\Phi=\Gamma(t)\left[x-x_{*}(t)\right], \\
P=P(t), \\
R=R(t) .
\end{gathered}
$$

Using this ansatz in $(5.8 \mathrm{a}, \mathrm{b})$ leads to

$$
\begin{gathered}
v_{1}=-\frac{\dot{\Gamma}}{\Gamma} x+\frac{\dot{\Gamma} x_{*}+\Gamma \dot{x}_{*}}{\Gamma}, \\
R \Gamma b_{1}=0 .
\end{gathered}
$$

We put aside the case $R=0$, which would lead to trivial results or very particular cases of the Bäcklund transform of the Burgers equation. With the choice $b_{1}=0$, $v_{1}$ must be on its own a solution of the Burgers equation. Moreover, the conditions $(5.9 \mathrm{a}, \mathrm{b}) \mathrm{read}$

$$
\begin{gathered}
P_{t}=-\left[P v_{1}\right]_{x}=\frac{\dot{\Gamma}}{\Gamma} P, \\
R_{t}=R v_{1 x}=-\frac{\dot{\Gamma}}{\Gamma} R .
\end{gathered}
$$


To integrate these equations, two cases must be considered. First, if $\dot{\Gamma}$ does not vanish, a detailed calculation shows that they imply $R=0$, a case already put aside. Secondly, if $\dot{\Gamma}$ vanishes, so do $P_{t}$ and $R_{t}$, and $P, R$ and $\Gamma$ are pure constants. The equation (5.36a) reduces to $v_{1}=\dot{x}_{*}$, which implies, for $v_{1}$ to be a solution of the Burgers equation, that $v_{1 t}=0$; thus $v_{1}=\dot{x}_{*}$ is a pure constant, while $x_{*}=v_{1} t+x_{0}$. The resulting new fields are

$$
\begin{gathered}
v=-2 \sigma \frac{P}{\Gamma} \frac{1}{\left(x-v_{1} t\right)-x_{0}}+v_{1}, \\
b=-2 \sigma \frac{R}{\Gamma} \frac{1}{\left(x-v_{1} t\right)-x_{0}} .
\end{gathered}
$$

In this last case, the Bäcklund transform produces the solution (2.5), with a unique simple pole for $v$ and $b$, but after application of a Galilean boost with velocity $v_{1}$.

We stop the hunting here, considering that we have proven that the set of nontrivial instances of the Bäcklund transform is not empty and that the transform itself is not without interest.

\section{Summary and comments}

We have studied the one-dimensional MHD system, proposed by J. H. Thomas, at very large magnetic Prandtl number. Specifically, we have concentrated on solutions where the small-scale excitation is localized in a small spatial region, just like shocks in ordinary hydrodynamics, or, to stay with one-dimensional models, like the shocks in the Burgers equation. In the Thomas system, the magnetic induction field and the velocity field are coupled so that there is a variety of localized solutions, which we have called defects, according to the terminology used in condensed matter physics. We have found that the location of the core of the defect is always the same for the two fields and that the spatial extension or width of this defect is always the same for these two fields. This is remarkable, since there is no length scale at one's disposal in the magnetic induction equation in the present limit of infinite Prandtl number. This result is particularly noteworthy, since a factor of proportionality different from one between the two length scales could have been possible.

We have studied numerically the generation and evolution of these defects. Some phenomenological rules have been drawn, such as (i) 'there is a preference for states where the magnetic energy is larger than the kinetic energy', which one may trace back to the absence of magnetic energy dissipation; or (ii), less obviously, in the shock defects, 'states where the velocity exhibits an antishock are preferred'. A mathematical rule has been obtained: 'in the presence of a magnetic oscillatory (bump/hollow) defect, the velocity exhibits a shock defect'. We believe that this set of predictions may be of interest for the physical interpretation of plasmas amenable to one-dimensional MHD modelling with large Reynolds and Prandtl numbers.

Finally, we want to stress that many phenomena could not have been explained without a proper understanding of the (spatial) analytic structure of the solutions. Theoretically, the formal existence of a functional Laurent-Painlevé expansion and of a Bäcklund transform marks the partially integrable character of the Thomas system with zero magnetic diffusivity. In practice, we have obtained all the 
travelling-wave solutions of the Thomas system, which all turn out to be meromorphic; they provide a complete description of the shock/antishock defects. Similarly, for the magnetic hollow defect, we have introduced meromorphic asymptotic representations. The two laws mentioned above concerning the position and extension of the defect have received in this way a simple interpretation in terms of the position of the poles of $v$ and $b$ in the (spatial) complex plane. Similarly, the forbidding of certain topologies of the defects corresponds to the lack of a solution for the equation linking the residues of the $v$-pole and the $b$-pole; while the existence of purely imaginary solutions to this same equation gave an explanation of the shape of the magnetic hollow wave. Every piece of knowledge that we had on the meromorphy properties of the solutions has thus proven useful, not only to interpret but also to explain some of the physics of the system.

\section{Acknowledgements}

We thank Th. Passot and A. Pouquet for discussions on their own work and on the present work. We thank G. Belmont, R. Grappin and M.C. Festou for correspondence on solar system plasmas. Some remarks by A.C. Newell stimulated us to pursue the study of the partially integrable character of the system, and $\mathrm{E}$. Delabaere helped us to understand the topology of the hyperboloid (2.12) in $\mathbb{C} \times \mathbb{C}$. A remark by A. Noullez concerning a numerical treatment of the data was very helpful. We thank H.H. Rugh for a useful suggestion. The (anonymous) referee had a very useful impact on the discussion of the validity of the assumptions made in deriving the model and its predictions. J.-D. F. acknowledges the hospitality of the Mathematics Institute at the University of Warwick. S. G. is supported by a grant from the EC (FMRX-CT98-0175).

\section{Appendix. Absence of a general solution to the $\tau$-function formalism}

This appendix is a complement to Sec. 5.2. From $(5.8 \mathrm{c})$ with $b_{1}=0$, we read off that $R^{2} / \Phi_{x}$ is a function of time $t$ only; we know from (5.20) that $R$ is a function of space $x$ only. These two pieces of information are gathered with the following notation:

$$
\begin{gathered}
\Phi(t, x)=L(t) G(x)+J(t) \\
G^{\prime}=R^{2},
\end{gathered}
$$

where $L(t)$ does not vanish identically. The prime indicates the derivative with respect to $x$. Using (5.20) and the IR written for $R$, one finds that $R \Phi_{t} / \Phi_{x}$ is a function of time $t$ only. This is embodied in the notation

$$
R \Phi_{t}=N(t) \Phi_{x}
$$

where $N(t)$ is a non-zero function of $t$. Combining with (A 1) yields

$$
\frac{\dot{L}}{L N}=\frac{R^{\prime}}{R^{2}}=C,
$$

where $C$ is a pure real constant and the dot indicates the derivative with respect to $t$.

If $C$ vanishes, then $L=L_{0}, R=R_{0}$ and $G^{\prime}=R_{0}^{2}$, where $L_{0}$ and $R_{0}$ are pure non-zero real constants. Consequently,

$$
\Phi=L_{0} R_{0}^{2}\left[x-x_{*}(t)\right] .
$$


With this notation, the dynamical equation (5.8a) yields

$$
P=-\frac{L_{0} R_{0}^{2}}{2 \sigma} \dot{x}_{*}(t) x+E(t)
$$

where $E(t)$ is a function of $t$ only. Combining the above remarks with (A 5), the SR becomes a polynomial of degree two in $x$ that has to vanish identically. This yields, in particular,

$$
\begin{gathered}
E=\frac{1}{2} L_{0} R_{0}^{2}, \\
E^{2}=R_{0}^{2}+L_{0} R_{0} E,
\end{gathered}
$$

which has no solution under the above hypotheses.

If $C$ is a non-zero real number, then (A 3) can be integrated non-trivially and yields

$$
\begin{gathered}
L(t)=L_{0} \exp \left(C \int N(s) d s\right), \\
R(x)=\frac{-1}{C} \frac{1}{x-x_{*}}, \\
\Phi(x, t)=L(t)\left(\frac{-1}{C^{2}} \frac{1}{x-x_{*}}+E\right)+J(t),
\end{gathered}
$$

where $C, L_{0}, x_{*}$ and $E$ are pure real constants, with $C \neq 0$ and $L_{0} \neq 0$. When used in (A 1) and (A 2), these expressions can be further simplified into

$$
\begin{gathered}
J=-E \dot{L}, \\
\Phi=L \frac{-1}{C^{2}} \frac{1}{x-x_{*}}+J_{0},
\end{gathered}
$$

where $J_{0}$ is a pure real constant. Using this last expression, one can integrate (5.8a) for $P$, which reads

$$
P=\frac{1}{x-x_{*}}\left(\frac{-\dot{L}}{2 \sigma C^{2}} x-\alpha(t)\right) .
$$

Finally, one collects all the pieces in the SR, which becomes again a second-degree polynomial in $x$; identifying the coefficients, one obtains $\dot{L}=0$, which contradicts $C \neq 0$.

\section{References}

Ablowitz, M. J., Ramani, A. and Segur, H. 1980 A connection between nonlinear evolution equations and ordinary differential equations of p-type I. J. Math. Phys. 21, 715-721.

Arnold, V. I. 1973 Ordinary Differential Equations. Moscow: Mir.

Bender, C. M. and Orszag, S. A. 1978 Advanced Mathematical Methods for Scientists and Engineers. McGraw Hill.

Bessis, D. and Fournier, J.-D. 1984 Pole condensation and the Riemann surface associated with a shock in Burgers' equation. J. Physique Lett. 45, L833-L841.

Bessis, D. and Fournier, J.-D. 1990 Complex singularities and the Riemann surface for the Burgers equation. In: Nonlinear Physics (ed. G. Chaohao, L. Yishen and T. Guizhang) Berlin: Springer-Verlag, pp. 252-257.

Bhattacharjee, A., Ng, C.S. and Spangler, S. R. 1998 Weakly compressible magnetohydrodynamic turbulence in the solar wind and the interstellar medium. Astrophys. J. $\mathbf{4 9 4}$ $409-418$. 
Brunelli, J. C. and Das, A. 1997 A Lax description for polytropic gas dynamics. Phys. Lett. A 235A, 597-602.

Burgers, J. M. 1939 Mathematical examples illustrating relations occurring in the theory of turbulent fluid motion. Kon. Ned. Akad. Wet. Verh. 17, 1-53.

Burgers, J. M. 1974 The Nonlinear Diffusion Equation. Dordrecht: Reidel.

Burlaga, L. F. 1991 Intermittent turbulence in the solar wind. J. Geophys. Res. 96, 5847-5851.

Chabat, B. 1990 Introduction à l'Analyse Complexe. Moscow: Mir.

Cole, J. 1951 On a quasi-linear parabolic equation occurring in aerodynamics. Q. Appl. Maths 9, 225-236.

Conte, R. and Boccara, N. (eds) 1990 Partially Integrable Evolution Equations in Physics. Dordrecht: Kluwer.

Dobrowolny, M., Mangeney, A. and Veltri, P. 1980 Properties of magnetohydrodynamic turbulence in the solar wind. Astron. Astrophys. 83, 26-32.

Festou, M. C., Rickman, H. and West, R. M. 1993 Comets. Astron. Astrophys. Rev. Part I 4, $363-447$.

Flaschka, H., Newell, A.C. and Tabor, M. 1991 Integrability, What is integrability? In: Nonlinear Dynamics (ed. V. E. Zakharov). Berlin: Springer-Verlag, pp. 73-114.

Fournier, J.-D. 1986 Propriétés locales et singularités complexes en dynamique non linéaíre. In: Méthodes Mathématiques pour l'Astrophysique (ed. M. Auvergue and A. Baglin). Nice: SFSA, pp. 333-383 (available from Dr D. Benest, Observatoire de Nice).

Fournier, J.-D. and Bessis, D. 1994 Dealing with the singularities of analytic functions. In: An Introduction to Methods of Complex Analysis and Geometry for Classical Mechanics and Non-Linear Waves (ed. D. Benest and C. Froesuhlé). Gif sur Yvette: Editions Frontières, pp. 1-45.

Fournier, J.-D. and Frisch, U. 1983 L'équation de Burgers déterministe et statistique. J. Méc. Théor. Appl. 2, 699-750.

Fournier, J.-D., Levine, G. and Tabor, M. 1988 Singularity clustering in the Duffing oscillator. J. Phys. A21, 33-54.

Fournier, J.-D., Spiegel, E.A. and Thual, O. 1989 Meromorphic integrals of two nonintegrable systems. In: Non Linear Dynamics (ed. G. Turchetti). Singapore: World Scientific, pp. 366-373.

Frisch, U. and Morf, R. 1981 Intermittency in nonlinear dynamics and singularities at complex times. Phys. Rev. A23, 2673-2705.

Galsgaard, K. and Nordlund, A. 1996 The heating and activity of the solar corona: boundary shearing of an initially homogeneous magnetic field. J. Geophys. Res. 101, 13445-13460.

Galtier, S. and Fournier, J.-D. 1998 Shocks and antishocks in the MHD-Thomas model In: Nonlinear Dynamics in the Heliosphere. Geophysical Research Abstracts (III), EGS Conference, Nice.

Galtier, S. and Pouquet, A. 1998 Solar flare statistics with a one-dimensional MHD model. Solar Phys. 179, 141-165.

Galtier, S., Nazarenko, S. V., Newell, A.C. and Pouquet, A. 2000 A weak turbulence theory for incompressible magnetohydrodynamics. J. Plasma Phys. 63, 447-488.

Goldstein, M. L. and Roberts, D. A. 1999 Magnetohydrodynamic turbulence in the solar wind. Phys. Plasmas 6, 4154-4160.

Gurbatov, S. N., Simdyankin, S. I., Aurell, E., Frisch, U. and Tóth, G. 1997 On the decay of Burgers turbulence. J. Fluid Mech. 344, 339-374.

Heiles, C., Goodman, A.A., McKee, C.F. and Zweibel, E. G. 1993 Magnetic fields in starforming regions : observations. In: Protostars and Planets III (ed. E. H. Levy. J. I. Lunine, M. Guerrieri and M. S. Matthews). Tucson: University of Arizona Press, pp. 279-326.

Hopf, E. 1950 The partial differential equation $u_{t}+u u_{x}=u_{x x}$. Commun. Pure Appl. Mech. 3, 201-230.

Kida, S. 1979 Asymptotic properties of Burgers turbulence. J. Fluid Mech. 93, 337-377.

Levine, G. and Tabor, M. 1988 Integrating the nonintegrable: analytic structure of the Lorenz system revisited. Physica D33, 189-210.

Marsch, E. and Tu, C. Y. 1994 Non-Gaussian probability distributions of solar wind fluctuations. Ann. Geophys. 12, 1127-1138. 
Newell, A. C. 1985 Solitons in Mathematics and Physics. Philadelphia: SIAM.

$\mathrm{Ng}$, C.S. and Bhattacharjee, A. 1997 Scaling of anisotropic spectra due to the weak interaction of shear-Alfvén wave packets. Phys. Plasmas 4, 605-610.

Olver, P. J. and Nutku, Y. 1988 Hamiltonian structures for systems of hyperbolic conservation laws. J. Math. Phys. 29, 1610-1619.

Parker, E. N. 1994 Spontaneous Current Sheets in Magnetic Fields. Oxford University Press.

Passot, T. 1986 Le test de Painlevé. In: Méthodes Mathématiques pour l'Astrophysique. (ed. M. Auvergne and A. Baglin). Nice: SFSA, pp. 161-170 (available from Dr D. Benest, Observatoire de Nice).

Passot, T. 1987 Simulations numériques d'écoulements compressibles. PhD Thesis, Université de Nice.

Passot, T. and Pouquet, A. 1986 The Painlevé analysis on the Burgers' MHD. Phys. Lett. 118, 121-123.

Passot, T. and Vázquez-Semadeni, E. 1998 Density probability distribution in onedimensional polytropic gas dynamies. Phys. Rev. E58, 4501-4510.

Pouquet, A., Galtier, S. and Politano, H. 1999 Mechanisms of injection and dissipation of energy and their relation to the dynamics of the interstellar medium. In: New Perspectives on the Interstellar Medium. (ed. A.R. Taylor, T.L. Landecker and G. Joncas). ASP Conference Series, Vol. 168, pp. 417-426.

Priest, E. R. 1982 Solar Magnetohydrodynamics. Dordrecht: Reidel.

Schwenn, R. and Marsch, E. 1991 Physics of the Inner Heliosphere II: Particles, Waves and Turbulence. Berlin: Springer-Verlag.

Shebalin, J. V., Matthaeus, W. H. and Montgomery, D. 1983 Anisotropy in MHD turbulence due to a mean magnetic field. J. Plasma Phys. 29, 525-547.

Sulem, C., Fournier, J.-D., Frisch, U. and Sulem, P. L. 1979 Remarques sur un modèle unidimensionnel pour la turbulence magnétohydrodynamique. C. R. Acad. Sci. Paris 288, $571-573$.

Sulem, C., Sulem, P. L. and Frisch, H. 1983 Tracing complex singularities with spectral methods. J. Comput. Phys. 50, 138-161.

Taylor, J. B. 1986 Relaxation and magnetic reconnection in plasmas. Rev.Mod.Phys. 58 $741-763$.

Thomas, J. H. 1968 Numerical experiments on a model system for magnetohydrodynamic turbulence. Phys. Fluids 11, 1245-1250.

Thomas, J. H. 1970 Model equations for magnetohydrodynamic turbulence. A gas dynamics analogy. Phys. Fluids 13, 1877-1879.

Weiss, J., Tabor, M. and Carnevale, G. 1983 The Painlevé property for partial differential equations. J. Math. Phys. 24, 522-526.

Wild, N., Gekelman, W. and Stenzel, R. L. 1981 Resistivity and energy flow in a plasma undergoing magnetic field line reconnection. Phys. Rev. Lett. 46, 339-342.

Zank, G.P. and Matthaeus, W. H. 1992 The equations of reduced magnetohydrodynamics J. Plasma Phys. 48, 85-100.

Zilbersher, D., Gedalin, M., Newbury, J. A. and Russell C.T. 1998 Direct numerical testing of stationary shock model with low Mach number shock observations. J. Geophys. Res. 103, 26775. 